E. RAMPONE ${ }^{1,2} \star$, A. W. HOFMANN ${ }^{2}$, G. B. PICCARDO ${ }^{1}$, R. VANNUCCI ${ }^{3,4}$, P. BOTTAZZI ${ }^{3}$ AND L. OTTOLINI ${ }^{3}$ 'DIPARTIMENTO DI SCIENZE DELLA TERRA, UNIVERSITÁ DI GENOVA, GORSO EUROPA 26, J6132 GENOVA, ITALY

'MAX-PLANCK-INSTITUT FÚR CHEMIE, POSTFACH 3060, 55020 MAINZ, GERMANY

3CNR-CENTRO DI STUDIO PER LA GRISTALlOCHIMICA E LA CRISTALlografia, VIA ABBIATEGRASSO 209,27 IOo PAVIA, ITALY

‘DIPARTIMENTO DI SCIENZE DELLA TERRA, UNIVERSITÁ DI PAVIA, VIA ABBIATEGRASSO 209, 27100 PAVIA, ITALY

\title{
Petrology, Mineral and Isotope Geochemistry of the External Liguride Peridotites (Northern Apennines, Italy)
}

Mantle peridotites of the External Liguride (EL) units (Northern Apennines) represent slices of subcontinental lithospheric mantle emplaced at the surface during early stages of rifting of the Jurassic Ligurian Piemontese basin. Petrological, ion probe and isotopic investigations have been used to unravel the nature of their mantle protolith and to constrain the timing and mechanisms of their evolution. EL peridotites are dominantly fertile spinel lherzolites partly recrystallized in the plagioclase lherzolite stability field. Clinopyroxenes stable in the spinel-facies assemblage have nearly flat REE patterns ( $C_{\mathrm{N}}$ ) $\left.S m_{\mathrm{N}}=0.6-0.8\right)$ at $(10-16) \times C l$ and high $\mathrm{Na}, \mathrm{Sr}, \mathrm{Ti}$ and $\mathrm{Zr}$ contents. Kaersutitic $-T_{i}$-pargasitic amphiboles also occur in the spinel-facies assemblage. Their LREE-depleted REE spectra and very low $S r, Z r$ and $B a$ contents indicate that they crystallized from hydrous fuids with low concentrations of incompatible elements. Thermometric estimates on the spinelfacies parageneses yield lithospheric equilibrium temperatures in the range $1000-1100^{\circ} \mathrm{C}$, in agreement with the stability of amphibole, which implies $\mathrm{T}<1100^{\circ} \mathrm{C}$. Sr and $\mathrm{Nd}$ isotopic compositions, determined on carefully handpicked clinopyroxene separates, plot within the depleted end of the MORB field ${ }^{87} \mathrm{Sr} /{ }^{86} \mathrm{Sr}=0.70222-0.70263 ; \quad{ }^{143} \mathrm{Nd} /{ }^{144} \mathrm{Nd}=0.513047-$ $0.513205)$ similar to many subcontinental orogenic spinel lherzolites from the western Mediterranean area (e.g. Ivrea Zone and Lanzo $\mathcal{N}$ ). The interpretation of the $E L$ lherzolites as subcontinental lithospheric mantle is reinforced by the occurrence of one extremely depleted isotopic composition $\left(^{87} \mathrm{Sr} /{ }^{86} \mathrm{Sr}=0.701736 ;{ }^{143} \mathcal{N d} /{ }^{144} \mathcal{N d}=0.513543\right)$. Sr and $\mathcal{N d}$ model ages, calculated assuming both CHUR and DM mantle sources, range between $2.4 \mathrm{Ga}$ and $780 \mathrm{Ma}$. In particular, the 1.2- $\mathrm{Ga} \mathrm{Sr}$ age and the 780-Ma $\mathrm{Nd}$ age can be regarded as minimum ages of differentiation. The transition from spinel- to plagioclase-facies assemblage, accompanied by progressive deformation (from granular to tectonite-mylonite textures), indicate that the EL lherzolites experienced a later, subsolidus decompressional evolution, starting from subcontinental lithospheric levels. $S m / N d$ isochrons on plagioclase-clinopyroxene pairs furnish ages of $\sim 165 \mathrm{Ma}$. This early Jurassic subsolidus decompressional history is consistent with uplift by means of denudation in response to passive and asymmetric lithospheric extension. This is considered to be the most suitable geodynamic mechanism to account for the exposure of huge bodies of subcontinental lithospheric mantle during early stages of opening of an oceanic basin.

\section{INTRODUGTION}

Mantle peridotites from collisional belts have been frequently considered as products of Phanerozoic, pre-orogenic, mid-ocean ridge processes (involving asthenosphere upwelling, partial melting and seafloor emplacement). However, petrological and geochemical studies have revealed that mantle rocks from Proterozoic to Phanerozoic subcontinental lithosphere are also represented within these associations (Menzies \& Dupuy, 1991).

Peridotites and ophiolites from the periMediterranean Alpine chains are related to the formation of the Jurassic Tethyan basin. Their different lithological associations are thought to be related to two different geotectonic settings: (1) a Red Sea type of rifting and ocean formation for the Western Mediterranean suites and (2) a subduction-related origin for most of the Eastern Mediterranean suites (Beccaluva et al., 1980, and references therein).

Mantle peridotites and ophiolites of the Western

* Corresponding author.

Present address: Dipartimento di Scienze della Terra,

Universitá di Genova, Corso Europa 26, 16ı32 Genova, Italy

(C) Oxford University Press 1995 
Alpine-Northern Apennine system are generally thought to have formed in the Jurassic LigurianPiemontese basin (JLPB), which separated the palaeo-European and Adriatic continental blocks. In the Northern Apennine orogenic belt, mantle peridotites and basaltic rocks occur in different struc-tural positions, with respect to both the original palaeogeographic settings and the primary relationships with the associated sedimentary sequences (Decandia \& Elter, 1969; Abbate et al., 1970; Pagani et al., 1972) (Fig. 1).

The Internal Liguride (IL) units are thought to have originated in an intra-oceanic setting. They contain depleted mantle peridotites, and basaltic intrusive and volcanic rocks, which represent the stratigraphic basement of the Upper Jurassic-Paleocene sedimentary sequences.

In the External Liguride (EL) units, fertile lherzolites and basalts occur mainly as huge olistoliths within the Cretaceous-Eocene flysch sequences, where they are associated with continental detritus (Hercynian felsic intrusives and volcanics, gneisses, micaschists and mafic granulites), which occurs in stratigraphic horizons directly overlying the ophiolites and belonging to the olistoliths proper (Eberhardt et al., 1962; Pagani et al., 1972).

Fertile spinel lherzolites, partly recrystallized in a plagioclase-facies assemblage, are characteristic of the EL units. Two alternative mechanisms are currently invoked for the exposure of fertile pl lherzolites: (1) active rifting, i.e. the adiabatic and diapiric upwelling of asthenospheric mantle (Nicolas, 1984, 1986); and (2) passive rifting, i.e. passive extension of the continental lithosphere with progressive upwelling, unroofing and exposure at the sea-floor of the lithospheric upper mantle (Decandia \& Elter, 1969; Piccardo, 1977; Lombardo \& Pognante, 1982; Beccaluva et al., 1984; Lemoine et al., 1986, 1987; Hoogerduijn Strating et al., 1990, 1993; Vissers et al., 1991).

Detailed petrological, geochemical and isotopic investigations have been performed on selected samples from the EL peridotites, with two main aims: (1) to constrain, by means of geochemical-isotopic and geochronologic evidence, the different evolutionary stages recorded by these peridotites; and (2) to assess whether this evolution is consistent with the uplift of subcontinental lithospheric mantle in response to pre-oceanic passive rifting.

\section{THE EXTERNAL LIGURIDE PERIDOTITE MASSIFS}

Within the Northern Apennines, the EL mantle peridotites crop out in two main localities: Suvero and Mt Aiona-Mt Nero-Mt Ragola (Elter \& Raggi, 1965; Pagani et al., 1972) (Fig. 1). In spite of widespread serpentinization, the EL ultramafic bodies preserve kilometre-scale volumes of unaltered peridotites in which mantle textures and assemblages are almost completely retained: the bodies mainly consist of spinel therzolites with isotropic, granular textures. Pyroxenite bands, mainly consisting of sp-bearing $\mathrm{Al}$-augite clinopyroxenites and $\mathrm{Cr}$-diopside websterites, are common within the peridotites. They are particularly abundant within the Suvero and Mt Aiona bodies. On the basis of petrographic and petrological studies, these pyroxenites have been interpreted (Piccardo, 1976; Rampone, 1987) as high-pressure cumulates from basaltic melts, in agreement with the interpretation of mafic layers in orogenic subcontinental-type peridotites and pyroxenite xenoliths in alkaline basalts (Bodinier et al., 1987; Menzies \& Hawkesworth, 1987; Fabriès et al., 1991).

Both peridotites and associated pyroxenites show effects of subsequent tectonic and metamorphic evolution, i.e. widespread partial recrystallization to plagioclase-bearing assemblages, and progressive deformation leading to porphyroclastic to granoblastic textures and tectonite to mylonite fabrics, occurred in kilometre-wide shear zones (Beccaluva et al., 1984; Piccardo et al., 1990; Rampone et al., 1993; and references therein). As a result, pyroxenite layers are strongly deformed and completely parallel to the peridotite foliation.

The EL peridotite-pyroxenite association was later intruded by gabbroic and late chilled basaltic dykes with MORB affinities (particularly abundant within the Mt Aiona massif) which crosscut most of the tectonite and mylonite fabrics (Rampone, 1987; Piccardo et al., 1990; Vannucci et al., 1993a).

\section{SAMPLES}

The investigated samples are lherzolites with significant modal amounts of clinopyroxene (up to 10$15 \%$ by volume). They were mainly collected at Suvero, Mt Nero and Mt Ragola, where the least serpentinized outcrops are found. Small-scale chemical heterogeneities in peridotites, possibly related to the pyroxenite intrusion, will not be addressed in this study. Therefore, we sampled only a few metre-scale homogeneous and massive rocks. They are spinel lherzolites showing different degrees of recrystallization under plagioclase-facies conditions and textures which range from granular to tectonite types. A 


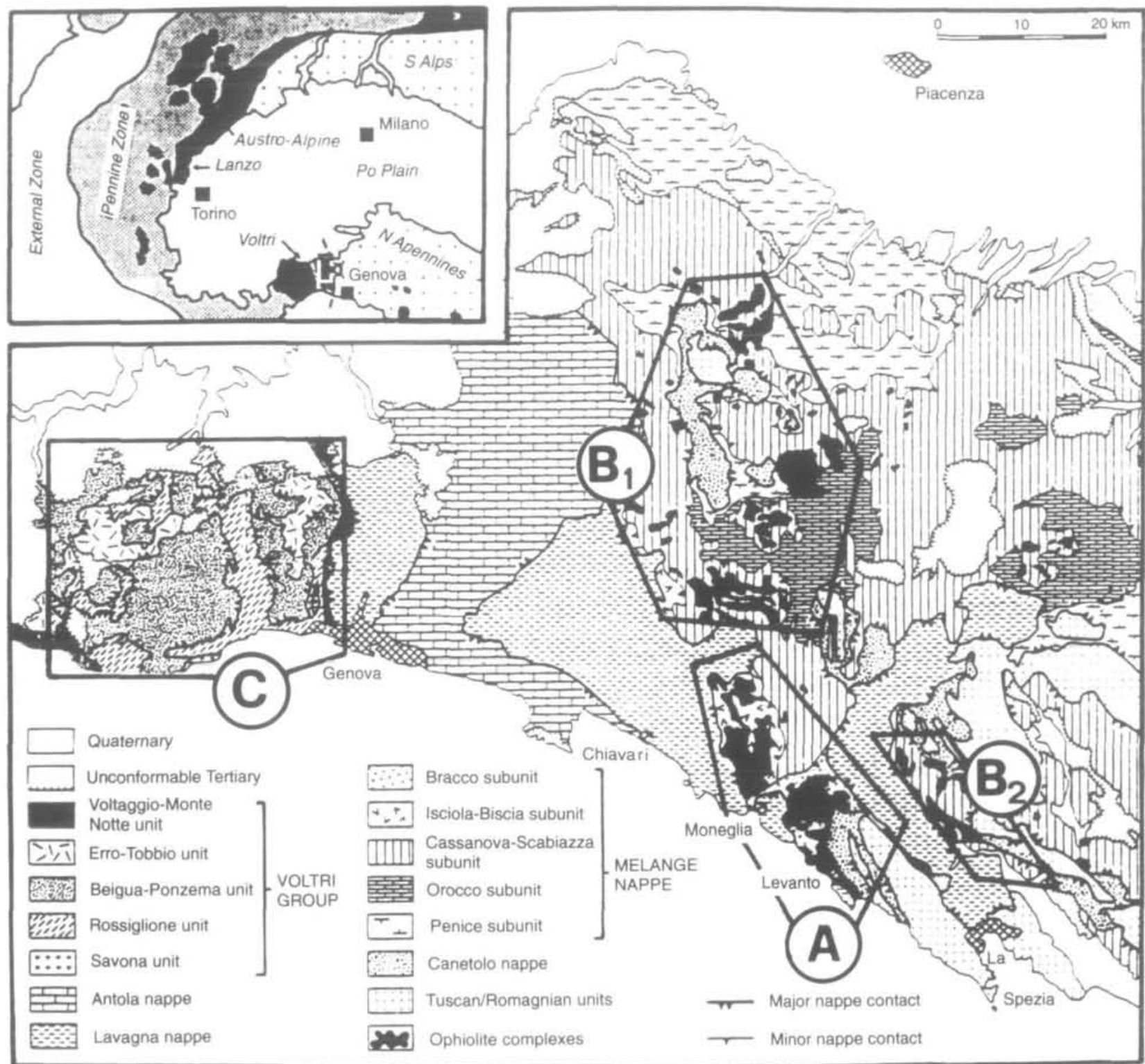

Fig. 1. Tectonic sketch map of the Ligurian Alps and Northern Apennines [redrawn after Hoogerduijn Strating (1991) and Hoogerduijn Strating et al. (1993)]. The ophiolitic sequences of the Northern Apennines, to which the peridotites studied belong, are the areas in black. A, Internal Liguride Units (IL); B , B , External Liguride Units (EL) (Mt Aiona-Mt Nero-Mt Ragola and Suvero areas, respectively); C, Voltri Massif, Ligurian Alps.

summary of the main petrographic and textural features of the investigated samples is given in Table 1.

In the sp lherzolites (Type A samples), the development of plagioclase is only incipient and mainly confined to (1) fine-grained granoblastic aggregates of $\mathrm{pl}+\mathrm{ol}+\mathrm{px}$, and (2) thin plagioclase rims around brown $\mathrm{Al}$-rich spinels. Moreover, they contain trace amounts of kaersutitic amphibole which is typically stable in the spinel facies. In the plagioclase-bearing assemblage, these amphiboles recrystallize to a $\mathrm{Ti}$ pargasitic composition. In the plagioclase lherzolites
(Type B samples), which constitute the predominant rock type, the plagioclase-facies recrystallization is well developed and manifested by (1) opx $+\mathrm{pl}$ exsolution within sp-facies clinopyroxene, (2) welldeveloped plagioclase rims around $\mathrm{Cr}$-rich spinels, and (3) granoblastic ol $+\mathrm{pl}+\mathrm{px}$ domains. Relics of the previous spinel-facies minerals are frequently preserved as porphyroclasts within the plagioclasebearing granoblastic matrix. Ti-pargasitic amphibole only occurs in the pl assemblage.

In the EL lherzolites, the metamorphic transition 
Table 1: Petrographic and textural features of investigated samples

\begin{tabular}{|c|c|c|c|}
\hline Rock type & Texture & Sample & Locality \\
\hline Type A & High strain tectonite. Highly deformed and elongated clino- and & ER-R3/3 & Mt Ragola \\
\hline Sp lherzolite with & orthopyroxenes. Tabular olivines with kink-banding. Elongated spinels & ER-S2/2 & Suvero \\
\hline incipient plagioclase & surrounded by thin plagioclase rims. Kaersutitic amphibole in equilibrium & ER-S2/1 & Suvero \\
\hline re-equilibration & $\begin{array}{l}\text { texture with the sp-facies assemblage. Narrow mylonitic bands } \\
\text { recrystallized to } \mathrm{pl}+\mathrm{ol}+\mathrm{Ti}-\mathrm{parg}+\mathrm{px}\end{array}$ & ER-S2/4 & Suvero \\
\hline Type B & (1) Ex-protogranular-porphyroclastic. Exsolved clino- and orthopyroxene & ER-N1/1 & Mt Nero \\
\hline Sp therzolite strongly & relics. Exsolutions (opx $+\mathrm{pl}$ ) better developed in cpx. Amoeboid dark & ER-N1/2 & Mt Nero \\
\hline re-equilbrated in & spinel porphyroclasts with well-developed plagioclase rims. Rare & ER-N1/3 & Mi Nero \\
\hline \multirow[t]{12}{*}{ pl-facies conditions } & triple-point equilibrium texture between opx and ol. Granoblastic domains & ER-N1/4 & Mt Nero \\
\hline & constituted by pl + ol + px. Small grains of Ti-pargasitic amphibole in & ER-N1/5 & Mt Nero \\
\hline & interstitial position between the porphyroclastic minerals, often showing & ER-N2/14 & Mi Nero \\
\hline & equilibrium texture with granoblastic ol and pl & ER-N2/16 & Mit Nero \\
\hline & & ER-N2/17 & Mi Nero \\
\hline & & ER-N2/18 & Mt Nero \\
\hline & & ER-R5/1 & Mt Ragola \\
\hline & & ER-R4/1 & Mt Ragola \\
\hline & (2) High-strain tectonite with recrystallized mylonitic bands. Tabular olivine. & ER-N2/7 & Mt Nero \\
\hline & Kink bands in olivine and pyroxenes. Elongated spinels with pl rims, often & ER-N2/1 & Mt Nero \\
\hline & dismembered along the tectonite foliation. Mylonitic bands recrystallized & ER-N2/2 & Mt Nero \\
\hline & to $p l+o l+p x+$ Ti-parg & SP4 & Suvero \\
\hline
\end{tabular}

from spinel- to plagioclase-facies stability field is recorded by significant within-mineral redistribution of both major and trace elements. This topic has already been discussed in previous papers (Beccaluva et al., 1984) and in a recent study (Rampone et al., 1993), which documents the trace element partitioning between mantle minerals during the spinelto plagioclase-facies reaction, based on electron and ion probe data from five selected samples. In this paper, we present a more complete data set of major and trace element data (on a larger number of samples), in addition to isotopic data. The major aim of this work is to discuss the primary compositional features of the peridotites, which are related to the complete spinel-facies equilibration, to unravel the nature of their mantle protolith.

\section{SAMPLE PREPARATION AND ANALYTIGAL PROCEDURES}

Whole-rock major and trace element compositions of $20 \mathrm{EL}$ peridotite samples were determined by conventional X-ray fluorescence (XRF) and inductively coupled plasma-atomic emission spectrometry (ICP-AES) techniques. Eight samples, representative of different sampling localities and of different paragenetic and textural types (see previous section), were analysed for major and trace element mineral composition and for $\mathrm{Sr}$ and $\mathrm{Nd}$ isotopic ratios.

Major element mineral analyses were performed by waveleng th-dispersive spectrometry (WDS) using an ARL SEMQ electron microprobe operating at 
$15 \mathrm{kV}$ (sample current $15 \mathrm{nA}$ ). Natural silicates and oxides were employed as standards. Data reduction was done according to the procedure of Bence \& Albee (1968). These analyses were carried out at the Centro di Studi per la Stratigrafia e la Petrografia delle Alpi Centrali, Dipartimento di Scienze della Terra, Milano.

In situ trace element mineral analyses (mainly on clinopyroxene and amphibole) were carried out with a Cameca IMS $4 \mathrm{f}$ ion-microprobe at GSCC Pavia. The 'energy filtering' technique (Shimizu et al., 1978) was employed to remove molecular interferences. The experimental set-up and quantification are similar to those described by Bottazzi et al. (1991). A detailed description of the specific analytical procedures has been reported by Rampone et al. (1993). Trace element abundances have been normalized to the average $\mathrm{Cl}$ chondrite composition (Anders \& Ebihara, 1982).

The isotope analyses were done at the MaxPlanck-Institut furr Chemie in Mainz. Most of the analyses were done on clinopyroxene separates. Typical sample weights were 20-30 mg. In samples ER-N2/16 and ER-N1/3, plagioclase has also been analysed, to test isotopic equilibrium between peridotite minerals and to obtain $\mathrm{pl}-\mathrm{cpx}$ isochrons. For most samples, both 'dirty' (direct output of the electromagnetic separator) and 'clean' (carefully handpicked) separates have been analysed. Both clean and dirty separates were subjected to several steps of leaching: (1) washing in $6 \mathrm{~N}$ double-distilled $\mathrm{HCl}$ in an ultrasonic bath for $20 \mathrm{~min}$; (2) leaching in hot $6 \mathrm{~N} \mathrm{HCl}$ for 2-3 h; (3) leaching in warm diluted $\mathrm{HNO}_{3}$; and (4) repeated washing with ultrapure water. As shown below in Table 11, no appreciable differences exist between isotopic ratios in dirty and clean separates. Consequently, they can be considered good duplicates.

The leached separates were spiked with a mixed ${ }^{150} \mathrm{Nd}^{-149} \mathrm{Sm}$ tracer, subsequently dissolved in $\mathrm{HF}-$ $\mathrm{HNO}_{3}$, and taken up in $6 \mathrm{~N} \mathrm{HCl}$. Sr was separated on a 5-ml AGV50W-X12 resin column. The separation of $\mathrm{Sm}$ and $\mathrm{Nd}$ from the REE was performed on 2-ml columns filled with HDEHP-coated Teflon powder, following the White \& Patchett (1984) procedure.

Isotopic analyses were carried out by a FinniganMAT 261 multicollector mass spectrometer. $\mathrm{Sr}$ was loaded with $\mathrm{TaF}_{5}$ onto $\mathrm{W}$ filaments. $\mathrm{Nd}$ was run in its metal form on Re filaments. During the period of analyses, several measurements of NBS $987 \mathrm{Sr}$ and La Jolla Nd standards gave the following values: ${ }^{143} \mathrm{Nd} /{ }^{144} \mathrm{Nd}=0.511833 \pm 21 \quad(n=18)$; ${ }^{87} \mathrm{Sr} /{ }^{86} \mathrm{Sr}=0.710238 \pm 18 \quad(n=23)$. Mass fractionations were corrected to ${ }^{146} \mathrm{Nd} /{ }^{44} \mathrm{Nd}=0.7219$ and
${ }^{86} \mathrm{Sr} /{ }^{88} \mathrm{Sr}=0 \cdot 1194$. Total procedural blanks were of the order 0.3-0.5 ng Sr, 0.01-0.1 ng Nd, 0.04 ng Sr.

The Sm-Nd isochrons were calculated using a value of $l_{\mathrm{Sm}}=6.54 \times 10^{-12} /$ year. Model ages were calculated using: $\left({ }^{143} \mathrm{Nd} /{ }^{144} \mathrm{Nd}\right)_{\text {CHUR }}=0.512638$; $\left({ }^{87} \mathrm{Sr} /{ }^{86} \mathrm{Sr}\right)_{\text {BSE }}=0.7047 ; \quad\left({ }^{147} \mathrm{Sm} /{ }^{144} \mathrm{Nd}\right)_{\mathrm{CHUR}}=$ $0.1967 ; \quad\left({ }^{87} \mathrm{Rb} /{ }^{86} \mathrm{Sr}\right)_{\mathrm{BSE}}=0.0868 ;{ }^{143} \mathrm{Nd} /{ }^{144} \mathrm{Nd}_{\mathrm{DM} 1}=$ $0.513114 ;{ }^{147} \mathrm{Sm} /{ }^{144} \mathrm{Nd} \mathrm{DM}_{\mathrm{DM}}=0.222 ;{ }^{143} \mathrm{Nd} /{ }^{144} \mathrm{Nd}_{\mathrm{DM} 2}$ $=0.51335$ (highest $\mathrm{Nd}$ isotopic ratio for present MORB; White, 1985 ); ${ }^{147} \mathrm{Sm} /{ }^{144} \mathrm{Nd}_{\mathrm{DM} 2}=0.2148$ (Hart \& Zindler, 1986); ${ }^{87} \mathrm{Sr} /{ }^{86} \mathrm{Sr}_{\mathrm{DM}}=0.7026$ (averaged value for MORB; Ito et al., 1987); ${ }^{87} \mathrm{Rb} /{ }^{86} \mathrm{Sr}_{\mathrm{DM}}=0.0509 ; l_{\mathrm{Rb}}=1.42 \times 10^{-11} /$ year (where CHUR, BSE and DM stand for chondritic uniform reservoir, bulk silicate Earth, and depleted mantle, respectively).

\section{WHOLE-ROCK GHEMISTRY}

Major and trace element concentrations of $20 \mathrm{EL}$ peridotites are listed in Table 2. In spite of widespread serpentinization, most of the analysed samples have LOI values $<5 \%$. Their fertile character (10-15 vol \% modal clinopyroxene) is confirmed by the relatively high $\mathrm{Al}_{2} \mathrm{O}_{3}(2 \cdot 86-4 \cdot 00$ wt $\%)$ and $\mathrm{CaO}(2.33-3.39$ wt \%) contents. Samples with the lowest $\mathrm{MgO}$ concentrations approach primitive mantle values (Ringwood, 1975; Jagoutz et al., 1979) (Figs 2 and 3). Such compositions are consistent with published whole-rock abundances for the EL peridotites (Ernst \& Piccardo, 1979; Ottonello et al., 1984).

The volatile-free compositions exhibit some welldefined chemical trends (Figs 2 and 3), consistent with compositional ranges for mantle peridotites described in the literature (Bonatti \& Michael, 1989; and references therein). Abundances of $\mathrm{CaO}$, $\mathrm{Al}_{2} \mathrm{O}_{3}, \mathrm{TiO}_{2}, \mathrm{~V}$ and $\mathrm{Sc}$ are inversely correlated with $\mathrm{MgO}$ contents. Similar trends have been reported for peridotites from Ronda, Horoman (see the compositional fields in Figs 2 and 3; Frey et al., 1985, 1991) and the Yugoslavian Dinaric Belt (Lugovic et al., 1991). However, the EL peridotites show a more limited range of variation of $\mathrm{MgO}$ abundances (38-41 wt \%). These trends are generally consistent with the primitive mantle estimates of Ringwood (1975) and Jagoutz et al. (1979). In fact, the primitive mantle values plot at the low$\mathrm{MgO}$ end of most correlations.

$\mathrm{Na}_{2} \mathrm{O}, \mathrm{Ni}$ and $\mathrm{Cr}$ concentrations of the analysed samples are not well correlated with $\mathrm{MgO}$ abundance. $\mathrm{Na}_{2} \mathrm{O}$ contents can be affected by significant analytical errors, owing to the very low concentrations. $\mathrm{Ni}$ and, to a minor extent, $\mathrm{Cr}$ contents are 
Table 2: Whole-rock major and trace element compositions for the External Liguride (EL) peridotites

\begin{tabular}{|c|c|c|c|c|c|c|c|c|c|}
\hline & ER-N1/1 & ER-N1/2 & ER-N1/3* & ER-N1 $/ 4^{\circ}$ & ER-N1/5 & ER-N2/2 & ER-N2/1 & SP4 & ER-S2/2 \\
\hline $\mathrm{SiO}_{2}$ & 42.57 & $42 \cdot 72$ & 43.07 & $42 \cdot 32$ & $42 \cdot 95$ & 40.92 & $41 \cdot 96$ & $42 \cdot 50$ & $42 \cdot 43$ \\
\hline $\mathrm{TiO}_{2}$ & 0.13 & 0.14 & 0.08 & 0.12 & 0.13 & 0.12 & 0.12 & 0.11 & 0.11 \\
\hline $\mathrm{Al}_{2} \mathrm{O}_{3}$ & 3.45 & $3 \cdot 29$ & 2.94 & $3 \cdot 47$ & $3 \cdot 37$ & $3 \cdot 29$ & 3.52 & 3.42 & 3.58 \\
\hline $\mathrm{Fe}_{2} \mathrm{O}_{3}$ & 8.75 & 9.08 & 8.78 & $8 \cdot 80$ & 8.88 & $8 \cdot 32$ & 8.23 & 8.29 & $8 \cdot 39$ \\
\hline Mno & 0.12 & 0.13 & 0.12 & 0.11 & 0.12 & 0.12 & 0.12 & 0.12 & 0.12 \\
\hline $\mathrm{MgO}$ & $37 \cdot 10$ & $39 \cdot 04$ & 39.54 & $37 \cdot 37$ & $38 \cdot 22$ & 36.50 & 36.65 & 37.00 & 36.97 \\
\hline $\mathrm{CaO}$ & $2 \cdot 87$ & 3.04 & $2 \cdot 36$ & $2 \cdot 83$ & $2 \cdot 91$ & $2 \cdot 74$ & 2.79 & $2 \cdot 86$ & $3 \cdot 22$ \\
\hline $\mathrm{Na}_{2} \mathrm{O}$ & 0.19 & 0.25 & 0.25 & 0.27 & 0.28 & 0.12 & 0.17 & 0.15 & 0.25 \\
\hline $\mathrm{H}_{2} \mathrm{O}^{+}$ & 5.03 & $1 \cdot 76$ & $2 \cdot 73$ & 3.92 & $2 \cdot 40$ & $7 \cdot 42$ & $6 \cdot 30$ & $5 \cdot 06$ & $4 \cdot 66$ \\
\hline Total & $100 \cdot 21$ & $99 \cdot 45$ & 99.87 & 99.21 & $99 \cdot 26$ & 99.55 & 99.86 & 99.51 & 99.83 \\
\hline $\mathrm{Ni}$ & 1959 & 1882 & 1828 & 1768 & 1769 & 1950 & 1853 & 1744 & 1891 \\
\hline Co & 107 & 92 & 91 & 82 & 84 & 121 & 100 & 77 & 97 \\
\hline Sc & 16 & 16 & 14 & 15 & 15 & 15 & 15 & 15 & 16 \\
\hline v & 77 & 74 & 59 & 72 & 70 & 69 & 70 & 65 & 78 \\
\hline $\mathrm{Cr}$ & 2773 & 2494 & 2498 & 2480 & 2354 & 2500 & 2750 & 2445 & 2664 \\
\hline $\mathrm{Cu}$ & 28 & 29 & 24 & 29 & 27 & 42 & 32 & 29 & 32 \\
\hline $\mathrm{Zn}$ & 51 & 51 & 46 & 50 & 49 & 47 & 51 & 46 & 49 \\
\hline $\mathrm{Zr}$ & 10 & 10 & 9 & 9 & 10 & 10 & 10 & 9 & 9 \\
\hline
\end{tabular}

Trace elements in p.p.m.

-Data from Rampone et al. (1991, 1993).

rather low with respect to normal abundances in peridotites with similar $\mathrm{MgO}$ contents. Again, this may be due to analytical errors. Moreover, the random $\mathrm{Cr}$ variation may reflect a non-uniform distribution of spinel, the main host phase for this element. The same possibility has been considered for the Horoman peridotites (Frey et al., 1991).

In Figs 2 and 3 , the compositional field for the Internal Liguride (IL, Northern Apennine) peridotites is also reported for comparison (Ottonello et al., 1984; Rampone, 1992). Relative to the EL samples, they show distinctly lower $\mathrm{CaO}, \mathrm{Al}_{2} \mathrm{O}_{3}, \mathrm{Na}_{2} \mathrm{O}$ and $\mathrm{TiO}_{2}$ contents. These depleted compositions are comparable with abyssal peridotites (Dick, 1989). Moreover, the Northern Apennine (EL and IL) peridotite compositional trends correspond well to the trends of the Ronda and Horoman ultramafics (Figs 2 and 3). This is especially clear for $\mathrm{CaO}$, $\mathrm{Al}_{2} \mathrm{O}_{3}$ and $\mathrm{TiO}_{2}$.

The element correlations defined by the EL peridotites are important in view of the recent con- troversy concerning the $\mathrm{Ca} / \mathrm{Al}$ ratio of the upper inantle. Palme \& Nickel (1985) found that the average $\mathrm{Ca} / \mathrm{Al}$ ratio of many sp lherzolite mantle xenoliths is $\sim 15 \%$ higher than in chondrites. Hart \& Zindler (1986) have subsequently suggested that the non-chondritic $\mathrm{Ca} / \mathrm{Al}$ ratio may be due to a sampling bias favouring cpx-rich rocks. More recently, Lugovic et al. (1991) have found a positive correlation of $\mathrm{MgO}$ vs $\mathrm{Ca} / \mathrm{Al}$ in peridotites from the Yugoslavian Dinaric Belt. They observed, however, that samples at the low- $\mathrm{MgO}$ end have chondritic $\mathrm{Ca} / \mathrm{Al}$ ratios. The $\mathrm{EL}$ peridotite data reinforce the observations of Lugovic et al. (1991) (Fig. 4). Most of the samples have lower $\mathrm{Ca} / \mathrm{Al}$ ratios than the xenoliths reviewed by Palme \& Nickel, and many have approximately chondritic ratios. Moreover, when the EL suite is combined with the peridotites of the Internal Ligurides (Rampone, 1992), a significant positive correlation becomes apparent, and the trend of this correlation line is consistent with a chondritic $\mathrm{Ca} / \mathrm{Al}$ ratio at primitive-mantle $\mathrm{MgO}$ values. 


\begin{tabular}{|c|c|c|c|c|c|c|c|c|c|c|}
\hline ER-S2/1 & ER-N2/14 & ER-N2/16 & ER-N2/17 & ER-N2/18 & ER-R5/1 & ER-R4/4 & ER-R4/1 & ER-S2/4 & ER-R3/3* & ER-N2/7 \\
\hline 41.90 & $42 \cdot 12$ & $43 \cdot 27$ & $41 \cdot 87$ & $42 \cdot 35$ & $42 \cdot 21$ & $41 \cdot 37$ & $42 \cdot 34$ & $42 \cdot 34$ & 43.19 & $41 \cdot 78$ \\
\hline 0.12 & 0.11 & 0.16 & 0.12 & 0.11 & $0 \cdot 10$ & $0 \cdot 13$ & 0.08 & 0.10 & 0.11 & 0.16 \\
\hline 3.55 & $3 \cdot 34$ & 4.00 & 3.50 & $2 \cdot 92$ & $3 \cdot 27$ & $3 \cdot 20$ & $2 \cdot 86$ & 3.08 & $3 \cdot 21$ & $3 \cdot 72$ \\
\hline 8.55 & $8 \cdot 55$ & 8.49 & 8.94 & $8 \cdot 78$ & 8.39 & $8-58$ & 8.64 & 8.64 & 8.49 & $8 \cdot 44$ \\
\hline 0.12 & 0.12 & 0.12 & 0.12 & 0.12 & 0.12 & 0.12 & 0.11 & 0.12 & 0.12 & 0.12 \\
\hline $36 \cdot 76$ & $37 \cdot 85$ & $36 \cdot 33$ & $37 \cdot 68$ & 38.84 & 37.59 & 36.87 & $38 \cdot 23$ & 38.93 & $38 \cdot 37$ & $35 \cdot 35$ \\
\hline $3 \cdot 17$ & 3.02 & $3 \cdot 19$ & 2.95 & $2 \cdot 75$ & $2 \cdot 62$ & $2 \cdot 79$ & $2 \cdot 33$ & $2 \cdot 40$ & 2.54 & $3 \cdot 39$ \\
\hline 0.20 & 0.25 & 0.41 & 0.20 & 0.20 & 0.24 & $0-20$ & 0.15 & 0.17 & 0.17 & 0.17 \\
\hline 4.84 & 4.52 & 3.53 & 3.84 & $4 \cdot 29$ & $5 \cdot 19$ & $5 \cdot 67$ & $4 \cdot 46$ & $4 \cdot 39$ & $4 \cdot 30$ & 5.89 \\
\hline $99 \cdot 30$ & $99-98$ & 99.50 & $99 \cdot 33$ & $100 \cdot 47$ & 99.81 & 99.04 & $99 \cdot 28$ & $100 \cdot 27$ & $100 \cdot 50$ & $99 \cdot 12$ \\
\hline 1982 & 2278 & 2027 & 1898 & 1965 & 1756 & 1858 & 1876 & 1981 & 1848 & 1747 \\
\hline 106 & 124 & 107 & 84 & 86 & 78 & 99 & 88 & 104 & 101 & 118 \\
\hline 16 & 16 & 17 & 16 & 15 & 15 & 15 & 13 & 14 & 14 & 17 \\
\hline 78 & 78 & 83 & 71 & 67 & 68 & 73 & 63 & 64 & 68 & 78 \\
\hline 2879 & 2724 & 2659 & 2549 & 2416 & 2511 & 2540 & 2868 & 2405 & 2673 & 2360 \\
\hline 28 & 25 & 35 & 28 & 28 & 24 & 30 & 21 & 23 & 28 & 32 \\
\hline 52 & 48 & 47 & 56 & 50 & 47 & 52 & 48 & 48 & 48 & 47 \\
\hline 9 & 11 & 13 & 11 & 10 & 10 & 11 & 9 & 8 & 10 & 13 \\
\hline
\end{tabular}

\section{MAJOR AND TRAGE} ELEMENT MINERAL

\section{GHEMIS TRY}

Representative major and trace element compositions of EL peridotite minerals are listed in Tables 3-10. Analyses have been performed on both spfacies (porphyroclastic) and pl-facies (granoblastic) minerals. As a whole, the major element mineral compositions presented here are consistent with previously published data (Beccaluva et al., 1984; Ernst \& Piccardo, 1979).

\section{Olivine}

Olivines have forsteritic compositions ( $\mathrm{Fo}_{89 \cdot 5-91 \cdot 0)}$. Their restricted compositional range is independent of grain-size or occurrence as porphyroclasts or granoblasts.

\section{Spinel}

Spinels vary considerably in both $\mathrm{Mg} /\left(\mathrm{Mg}+\mathrm{Fe}^{2+}\right)$ $(0.52-0.81)$ and $\mathrm{Cr} /(\mathrm{Cr}+\mathrm{Al})(0.07-0.47)$ (Fig. 5).
Rampone et al. (1993) demonstrated that this is a metamorphic compositional trend from Al-rich spinels in sp lherzolites (samples ER-R3/3 and ER-S2/2) to Cr-rich spinel relics in pl lherzolites (Type B samples). In fact, in spite of the large compositional variations recorded by the spinels, the bulk compositions of these samples are very similar. Al-rich spinels of sp lherzolite samples display compositions comparable with those of some subcontinental fertile sp lherzolites from the Alpine chain (e.g. Erro-Tobbio, Voltri Massif; Piccardo et al., 1990; Hoogerduijn Strating et al., 1993; Baldissero, Ivrea Zone; Rivalenti et al., 1979) (Fig. 5). They are, moreover, significantly different from spinel compositions of IL depleted peridotites (Beccaluva et al., 1984; Rampone, 1992), showing high $\mathrm{Cr}$ and low Al contents.

\section{Glinopyroxene}

Spinel-facies clinopyroxenes are Cr-bearing diopsides and are characterized by higher $\mathrm{Al}, \mathrm{Na}$ and lower $\mathrm{Cr}$ contents (Fig. 6) relative to plagioclasefacies clinopyroxenes. This is particularly evident in 

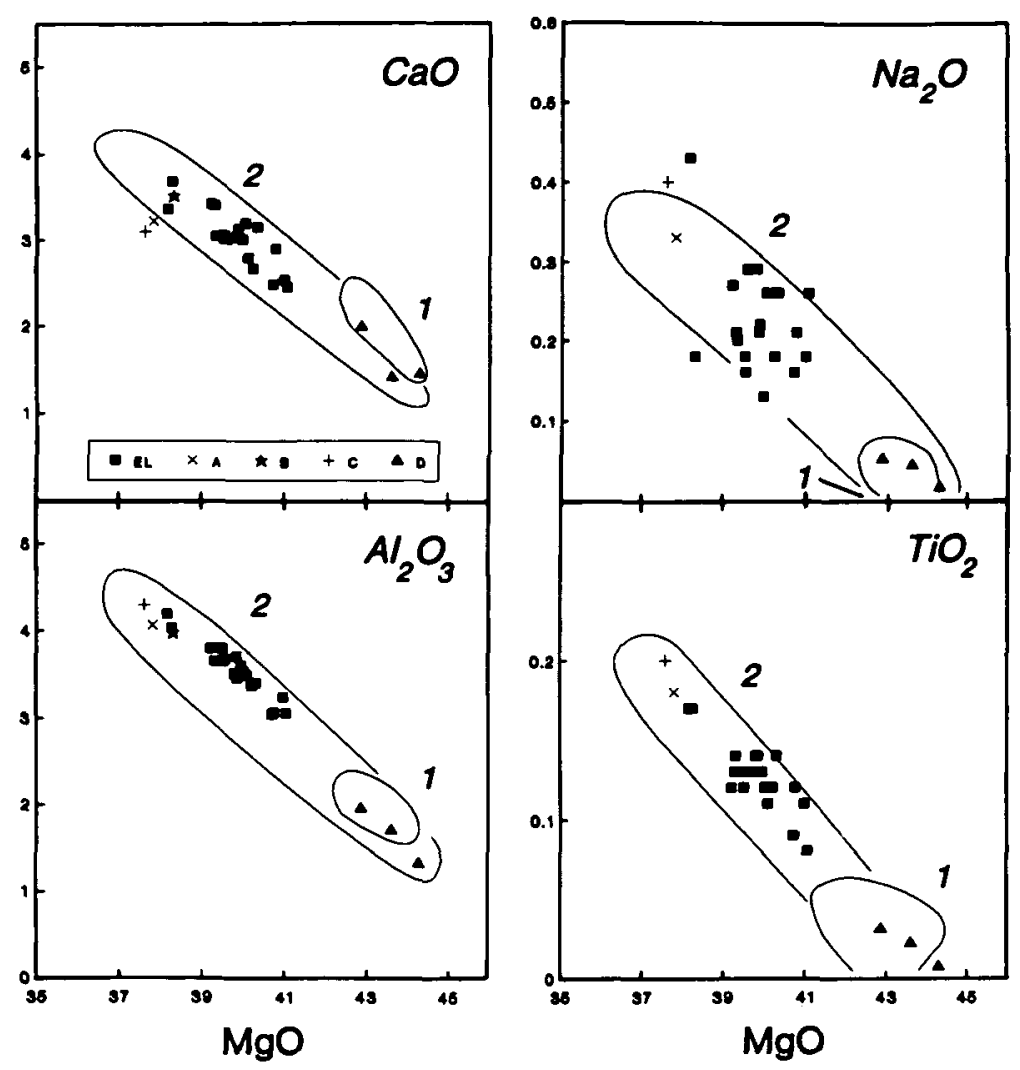

Fig. 2. Whole-rock abundances of $\mathrm{CaO}$, $\mathrm{Al}_{2} \mathrm{O}_{3}, \mathrm{Na}_{2} \mathrm{O}$ and $\mathrm{TiO}_{2}$ vs $\mathrm{MgO}$ (all data on anhydrous basis in wt \%). Symbols in the legend inside are as follows: EL, External Liguride peridotites; A, B, C, primordial mantle estimates from Hofmann (1988), Jagoutz et al. (1979) and Ringwood (1975) (pyrolite III), respectively; $D$, representative oceanic abyssal peridotite composition (Dick, 1989). The fields refer to the composition of peridotites from: (1) Internal Liguride (IL) Units, Northern Apennines (data from Rampone, 1992), (2) Ronda (data from Frey et al. (1985) and Horoman (data from Frey et al., I99I).
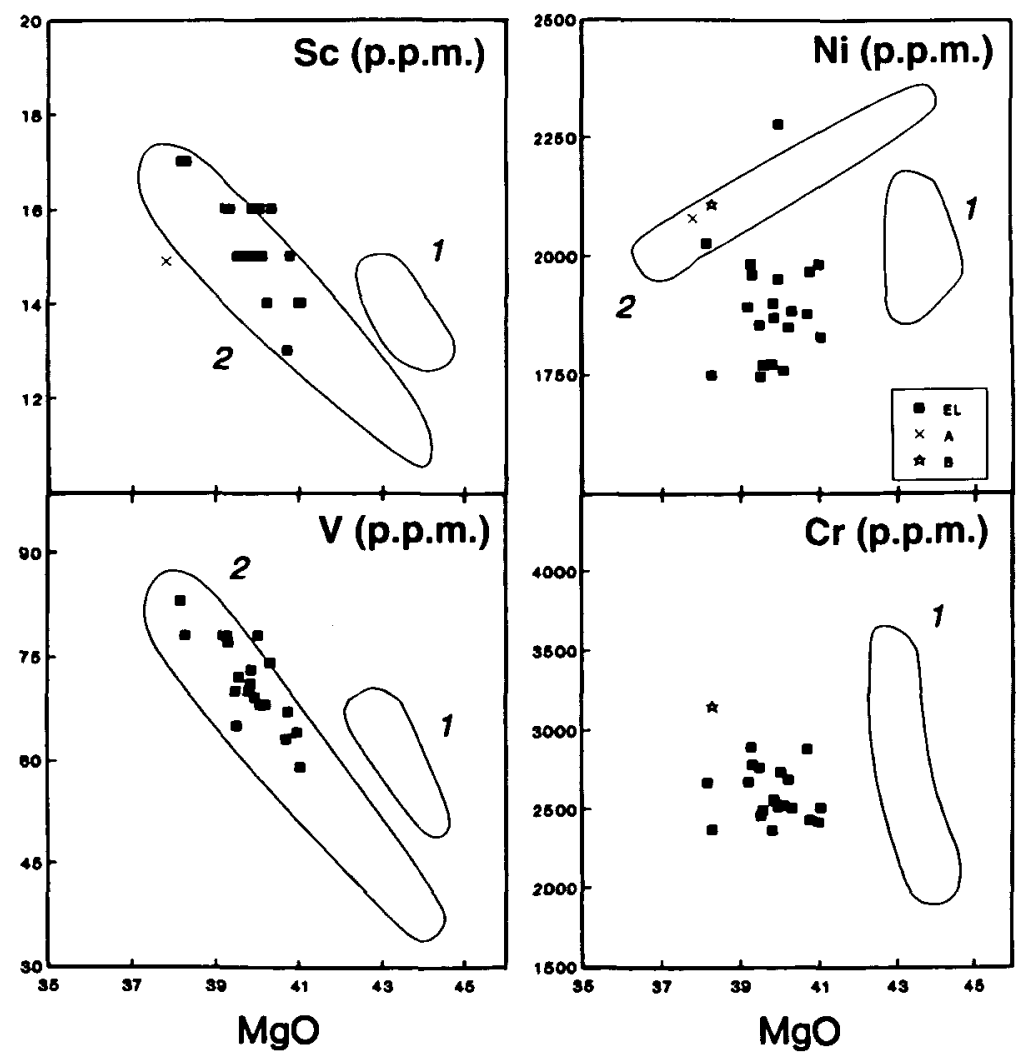

Fig. 3. Whole-rock abundances (p.p.m.) of $\mathrm{Sc}, \mathrm{V}, \mathrm{Ni}$ and $\mathrm{Cr}$ vs $\mathrm{MgO}$ (wt \%). Symbols and fields as in Fig. 2. 


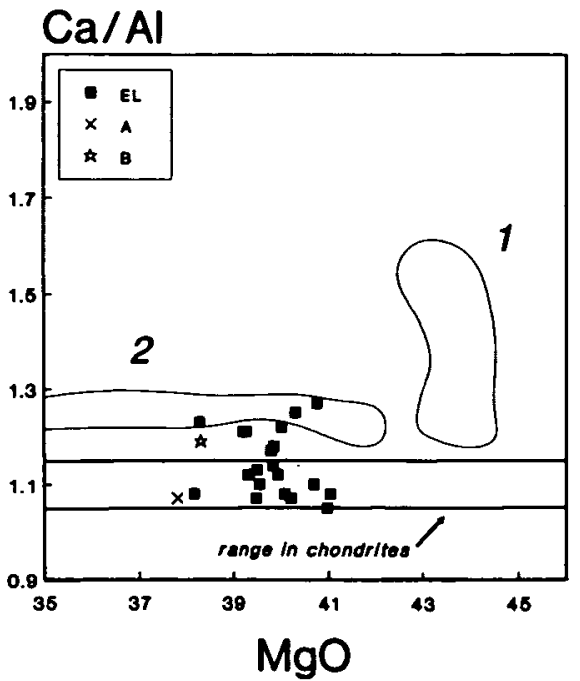

Fig. 4. $\mathrm{Ca} / \mathrm{Al}$ ratio vs $\mathrm{MgO}$ in the EL peridotites. Symbols as in Fig. 2. The field for range in chondrites is from Palme \& Nickel (1985). Other fields refer to: (1) IL peridotites (Rampone, 1992); (2) sp lherzolite mantle xenoliths studied by Palme \& Nickel (1985).

Type B samples, which record systematic compositional variations ( $\mathrm{Al}$ and $\mathrm{Na}$ decrease, and $\mathrm{Cr}$ increase) from core to rim in porphyroclasts, to granoblastic grains recrystallized in the plagioclasefacies assemblage (Rampone et al., 1993). The highest contents of $\mathrm{Al}\left(\mathrm{Al}_{2} \mathrm{O}_{3}\right.$ up to $\left.7-8 \mathrm{wt} \%\right)$ and $\mathrm{Na}\left(\mathrm{Na}_{2} \mathrm{O}\right.$ up to $\left.2 \mathrm{wt} \%\right)$ are recorded by clinopyroxene porphyroclasts in ER-R $3 / 3$ (the least pl- re-equilibrated sample). This feature is probably related to the lack of plagioclase exsolution in ERR3/3 clinopyroxene porphyroclasts, which are different in this respect from all the other samples studied.
Similarly to spinels, spinel-facies clinopyroxenes have compositions comparable with those of the Erro-Tobbio and Baldissero undepleted lherzolites (see Fig. 6). By contrast, clinopyroxenes from the IL peridotites (see compositional field in Fig. 6) exhibit significantly lower $\mathrm{Na}$ and higher $\mathrm{Cr}$ contents. Clinopyroxenes have nearly flat REE patterns, at $\sim(10-25) \times \mathrm{Cl} \quad\left(\mathrm{Ce}_{\mathcal{N}} / \mathrm{Yb}_{\mathcal{N}}=0.58-1.24 ; \quad \mathrm{Sm}_{\mathcal{N}} / \mathrm{Nd}_{\mathcal{N}}=\right.$ 0.96-1.25) (Fig. 7). Similar REE patterns are common in undepleted subcontinental-type spinel lherzolites from various environments such as xenoliths, orogenic massifs and pre-oceanic rifts (Frey \& Prinz, 1978; Stosch, 1982; Bodinier et al., 1988; Fabriès et al., 1989; Vannucci et al., 1991). The slight LREE depletion in clinopyroxenes is also consistent with published REE bulk-rock compositions of EL peridotites (Ottonello et al., 1984) (Fig. 7a). The lowest absolute REE concentrations $[(10-12) \times \mathrm{C} 1]$ are shown by clinopyroxene porphyroclasts in Type A samples. It is noticeable that the cores of sp-facies porphyroclasts in samples ER-R3/3 and ER-S2/2 display slightly positive $\mathrm{Eu}_{\mathcal{N}}$ anomalies (Fig. 7), which disappear towards the rims. By contrast, most of the clinopyroxenes in Type B samples are characterized by higher REE abundances [up to (2530) $\times \mathrm{Cl}$ in samples ER-N1/2 and ER-N1/5], and the development of slightly negative $\mathrm{Eu}_{N}$ anomalies. Moreover, clinopyroxene porphyroclasts in sample ER-R3/3 exhibit high $\mathrm{Sr}$ contents (55-65 p.p.m.), in agreement with their high $\mathrm{Na}$ and $\mathrm{Al}$ concentrations. Their $\mathrm{Zr}, \mathrm{Y}, \mathrm{Ti}, \mathrm{V}$ and $\mathrm{Sc}$ contents are 45-52 p.p.m., 25-30 p.p.m., 3500-3700 p.p.m., 210-220 p.p.m. and 52-62 p.p.m., respectively (Fig. 8a). These compositional ranges are typical for clinopyroxenes from undepleted sp lherzolites (O'Reilly et al., 1991; Vannucci et al., 1991). On the other hand,

Table 3: Major element compositions of olivines

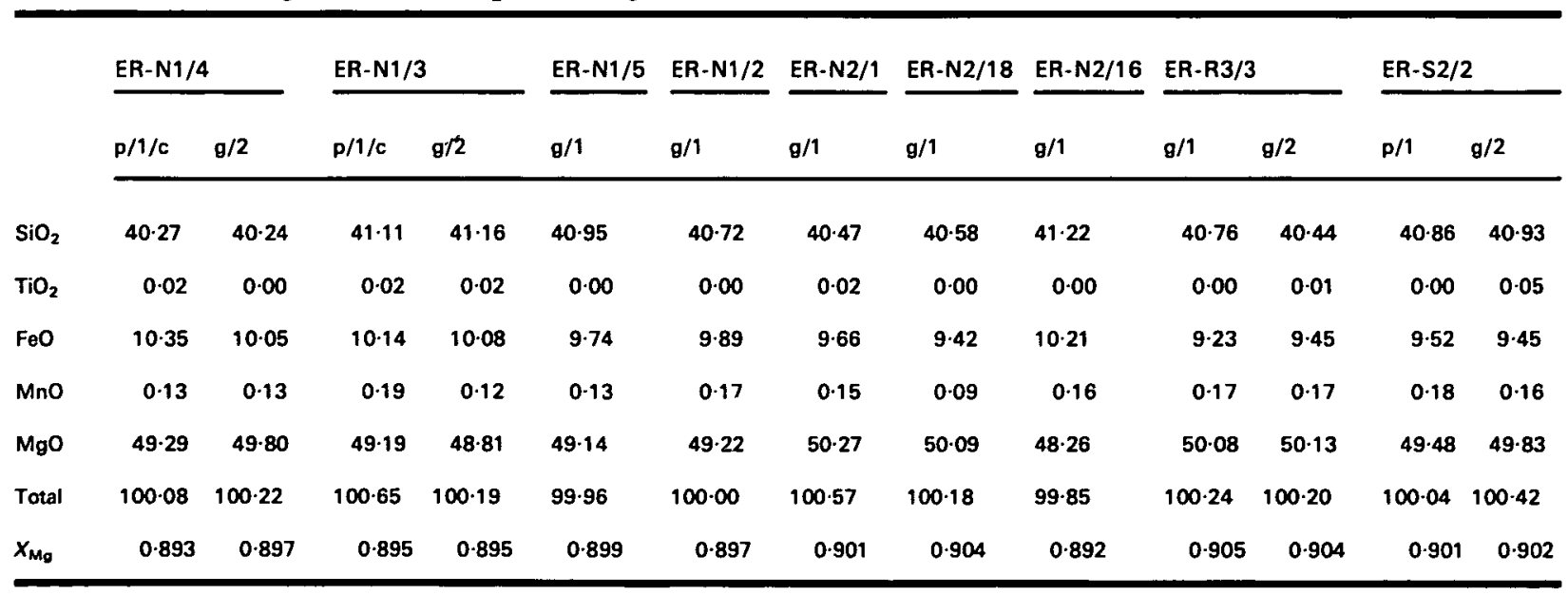

p, porphyroclast; g, granoblast; c, core. 
Table 4: Major element compositions of spinels

\begin{tabular}{|c|c|c|c|c|c|c|c|c|c|c|c|c|c|}
\hline & \multirow{2}{*}{$\frac{E R \cdot N 1 / 4}{c / 1^{*}}$} & \multirow{2}{*}{$\frac{E R-N 1 / 3}{c / 1^{*}}$} & \multirow{2}{*}{$\frac{\mathrm{ER}-\mathrm{N} 1 / 5}{\mathrm{c} / 1}$} & \multirow{2}{*}{$\frac{\mathrm{ER}-\mathrm{N} 1 / 2}{\mathrm{c} / 1^{\circ}}$} & \multicolumn{2}{|c|}{ ER-N2/1 } & \multicolumn{2}{|c|}{ ER-N2/18 } & \multirow{2}{*}{$\frac{E R-N 2 / 16}{c / 1}$} & \multicolumn{2}{|c|}{ ER-R3/3 } & \multicolumn{2}{|c|}{ ER-S2/2 } \\
\hline & & & & & $c / 1$ & $r / 1$ & $c / 1$ & $r / 1$ & & $\mathrm{c} / 1^{\bullet}$ & $r / 1^{\circ}$ & $\mathrm{c} / 1^{\bullet}$ & $r / 1^{*}$ \\
\hline $\mathrm{TiO}_{2}$ & 0.55 & 0.66 & 0.59 & 0.54 & 0.27 & 0.55 & 0.47 & 0.33 & 0.39 & 0.11 & 0.12 & 0.24 & 0.22 \\
\hline $\mathrm{Al}_{2} \mathrm{O}_{3}$ & $32 \cdot 19$ & $35 \cdot 88$ & $40 \cdot 32$ & $42 \cdot 32$ & 43.90 & 40.03 & $39 \cdot 48$ & $38 \cdot 86$ & $40 \cdot 34$ & $61 \cdot 80$ & 60.59 & 52.64 & 48.57 \\
\hline $\mathrm{Cr}_{2} \mathrm{O}_{3}$ & 31.00 & 29.01 & 25.44 & $22 \cdot 89$ & $22 \cdot 61$ & $26 \cdot 18$ & 26.54 & $27 \cdot 48$ & $24 \cdot 60$ & $6 \cdot 80$ & 8.02 & $15 \cdot 62$ & 19.08 \\
\hline $\mathrm{FeO}$ & $23 \cdot 36$ & $19 \cdot 19$ & 16.89 & $17 \cdot 77$ & $16 \cdot 26$ & $17 \cdot 70$ & $17 \cdot 23$ & $17 \cdot 45$ & $17 \cdot 35$ & 10.60 & 10.81 & $12 \cdot 68$ & $13 \cdot 19$ \\
\hline Mno & 0.22 & 0.20 & 0.18 & nd & 0.13 & 0.19 & 0.16 & 0.19 & 0.00 & 0.06 & 0.09 & 0.07 & 0.19 \\
\hline $\mathrm{MgO}$ & $13 \cdot 13$ & 15.92 & 16.98 & 16.99 & 17.09 & 15.98 & $16 \cdot 47$ & 16.19 & $16 \cdot 74$ & $21 \cdot 12$ & 20.84 & $19 \cdot 48$ & $18 \cdot 80$ \\
\hline Total & $100 \cdot 45$ & 100.86 & $100 \cdot 40$ & 100.51 & $100 \cdot 26$ & $100 \cdot 63$ & $100 \cdot 35$ & 100.50 & $99 \cdot 42$ & 100.49 & $100 \cdot 47$ & $100 \cdot 73$ & 100.05 \\
\hline
\end{tabular}

c, core; r, rim. `Data from Rampone et al. (1993).

Table 5: Major element compositions of clinopyroxenes

\begin{tabular}{|c|c|c|c|c|c|c|c|c|c|c|c|c|}
\hline & \multicolumn{3}{|l|}{ ER-N1/4 } & \multicolumn{3}{|c|}{ ER-N1/3 } & \multicolumn{2}{|c|}{ ER-N1/5 } & \multicolumn{3}{|c|}{ ER-N1/2 } & \multirow{2}{*}{$\frac{\text { ER-N2/ }}{p / 1 / c}$} \\
\hline & $p / 1 / c^{*}$ & $p / 1 / r^{*}$ & $g / 2^{*}$ & $p / 1 / c^{*}$ & $\mathrm{p} / 1 / \mathrm{r}^{\circ}$ & $g / 2^{*}$ & $p / 1 / c$ & $g / 2$ & $p / 1 / c^{\bullet}$ & $p / 1 / r^{\bullet}$ & $g / 2^{\circ}$ & \\
\hline $\mathrm{SiO}_{2}$ & $50 \cdot 36$ & $50 \cdot 60$ & $51 \cdot 20$ & 50.52 & $50 \cdot 75$ & 51.63 & 50.03 & $51 \cdot 30$ & 48.00 & $49 \cdot 40$ & $50 \cdot 10$ & $49 \cdot 60$ \\
\hline $\mathrm{TiO}_{2}$ & 0.91 & 0.98 & 0.91 & 0.65 & 0.74 & 0.77 & $0-98$ & 0.95 & 0.85 & $1 \cdot 13$ & $1 \cdot 17$ & 0.81 \\
\hline $\mathrm{Al}_{2} \mathrm{O}_{3}$ & 6.64 & $5 \cdot 52$ & $4 \cdot 82$ & $6 \cdot 32$ & $5 \cdot 72$ & $4 \cdot 35$ & $7 \cdot 38$ & $5 \cdot 61$ & 6.95 & $5 \cdot 19$ & 4.94 & 7.55 \\
\hline $\mathrm{Cr}_{2} \mathrm{O}_{3}$ & 0.86 & 0.99 & 1.09 & 1.15 & 1.09 & $1 \cdot 14$ & 0.85 & 0.92 & 0.87 & $1 \cdot 12$ & 0.95 & 0.84 \\
\hline $\mathrm{FeO}$ & 3.02 & $2 \cdot 99$ & 3.07 & $3 \cdot 33$ & 2.79 & 2.93 & 3.05 & 3.02 & $3 \cdot 19$ & $2 \cdot 74$ & $2 \cdot 83$ & $3 \cdot 00$ \\
\hline MnO & 0.11 & 0.06 & 0.12 & 0.07 & 0.11 & 0.04 & 0.09 & 0.04 & 0.26 & 0.27 & 0.21 & 0.05 \\
\hline $\mathrm{MgO}$ & $15 \cdot 80$ & $16 \cdot 16$ & 16.39 & 16.03 & $15 \cdot 56$ & $16 \cdot 30$ & $15 \cdot 29$ & $15 \cdot 51$ & $15 \cdot 69$ & $16-35$ & $16 \cdot 25$ & $15 \cdot 14$ \\
\hline $\mathrm{CaO}$ & 22.42 & $22 \cdot 34$ & $22 \cdot 33$ & $21 \cdot 77$ & $22-72$ & $22-10$ & 22.39 & $22 \cdot 21$ & 22.58 & $23 \cdot 11$ & 22.99 & $22 \cdot 26$ \\
\hline $\mathrm{Na}_{2} \mathrm{O}$ & 0.77 & 0.69 & 0.65 & 0.68 & 0.66 & 0.64 & 0.82 & 0.72 & 0.81 & 0.67 & 0.70 & 0.80 \\
\hline $\mathrm{K}_{2} \mathrm{O}$ & 0.00 & 0.00 & 0.00 & 0.00 & 0.00 & 0.00 & 0.00 & 0.01 & 0.00 & 0.00 & 0.07 & 0.00 \\
\hline \multirow[t]{3}{*}{ Total } & $100 \cdot 69$ & $100 \cdot 33$ & $100 \cdot 36$ & $100 \cdot 02$ & $100 \cdot 14$ & 99.90 & $100 \cdot 88$ & $100 \cdot 29$ & $100 \cdot 10$ & 99.98 & $100 \cdot 21$ & $100 \cdot 05$ \\
\hline & \multicolumn{2}{|l|}{ ER-N2/1 } & \multicolumn{2}{|c|}{ ER-N2/18 } & \multicolumn{3}{|c|}{ ER-N2/16 } & \multicolumn{3}{|l|}{ ER-R3/3 } & \multicolumn{2}{|l|}{ ER-S2/2 } \\
\hline & $p / 1 / r$ & $g / 2$ & $p / 1 / c$ & $g / 2$ & $\mathrm{p} / 1 / \mathrm{c}$ & $\mathrm{p} / 1 / \mathrm{r}$ & $g / 2$ & $\mathrm{p} / 1 / \mathrm{c}^{\bullet}$ & $p / 1 / r^{*}$ & $g / 2^{\circ}$ & $p / 1 / c^{*}$ & $g / 2^{*}$ \\
\hline $\mathrm{SiO}_{2}$ & $50 \cdot 81$ & $51 \cdot 73$ & 51.00 & $51 \cdot 45$ & 50.98 & 51.09 & $51 \cdot 34$ & 51.75 & $51 \cdot 32$ & $51 \cdot 18$ & 49.98 & $52 \cdot 50$ \\
\hline $\mathrm{TiO}_{2}$ & 1.00 & 0.80 & 0.98 & 0.81 & 0.89 & 0.88 & 0.79 & 0.65 & 0.69 & 0.93 & 0.57 & $0 \cdot 70$ \\
\hline $\mathrm{Al}_{2} \mathrm{O}_{3}$ & 5.42 & $3 \cdot 88$ & $5 \cdot 96$ & $4 \cdot 50$ & $5 \cdot 86$ & 4.71 & 4.83 & $8 \cdot 14$ & 8.01 & $5 \cdot 77$ & $7 \cdot 50$ & $4 \cdot 88$ \\
\hline $\mathrm{Cr}_{2} \mathrm{O}_{3}$ & 0.88 & 0.84 & 0.96 & 1.15 & 1.09 & 1.11 & 1.32 & 0.60 & 0.54 & 0.71 & 0.78 & 0.77 \\
\hline $\mathrm{FeO}$ & $2 \cdot 87$ & $2 \cdot 82$ & 2.90 & 3.57 & 2.84 & 3.03 & 3.64 & 2.58 & $2 \cdot 63$ & 2.55 & $2 \cdot 76$ & 2.94 \\
\hline Mno & 0.12 & 0.06 & 0.11 & 0.09 & 0.05 & 0.08 & 0.14 & 0.18 & 0.05 & 0.07 & 0.14 & 0.14 \\
\hline $\mathrm{MgO}$ & $15 \cdot 89$ & 16.90 & $15 \cdot 84$ & 16.58 & 15.01 & $15 \cdot 70$ & $15 \cdot 31$ & $14 \cdot 10$ & 14.45 & $15 \cdot 81$ & 14.96 & 16.97 \\
\hline $\mathrm{CaO}$ & $22 \cdot 37$ & 22.84 & $22-40$ & $21-88$ & $22 \cdot 27$ & $22-03$ & 22.08 & 20.49 & 21.00 & $22 \cdot 72$ & $22 \cdot 31$ & 21.05 \\
\hline $\mathrm{Na}_{2} \mathrm{O}$ & 0.71 & 0.62 & 0.73 & 0.59 & 0.80 & 0.88 & 0.48 & 1.97 & 1.66 & 0.84 & 0.94 & 0.70 \\
\hline $\mathrm{K}_{2} \mathrm{O}$ & 0.00 & 0.00 & 0.01 & 0.00 & 0.00 & 0.00 & 0.00 & 0.00 & 0.00 & 0.00 & 0.11 & 0.00 \\
\hline Total & $100 \cdot 07$ & $100 \cdot 49$ & $100 \cdot 89$ & $100 \cdot 62$ & 99.79 & 99.51 & 99.93 & $100 \cdot 46$ & $100 \cdot 35$ & 100.03 & 100.05 & $100 \cdot 65$ \\
\hline
\end{tabular}

p, porphyroclast; $g$, granoblast; c, core; r, rim.

-Data from Rampone et al. (1993). 
Table 6: Major element compositions of orthopyroxenes

\begin{tabular}{|c|c|c|c|c|c|c|c|c|c|c|}
\hline & \multicolumn{3}{|c|}{ ER-N1/4 } & \multicolumn{2}{|c|}{ ER-N1/3 } & \multicolumn{3}{|l|}{ ER-N1/5 } & \multirow{2}{*}{$\begin{array}{l}\text { ER-N1/2 } \\
p / 1 / c^{*}\end{array}$} & \multirow{2}{*}{$\frac{\text { ER-N2/1 }}{p / 1 / c}$} \\
\hline & $p / 1 / c^{*}$ & $p / 1 / r^{*}$ & $g / 2$ & $p / 1 / c^{\bullet}$ & $p / 1 / r^{*}$ & $p / 1 / c$ & $g / 2 / e x$ & $g / 3$ & & \\
\hline $\mathrm{SiO}_{2}$ & $53 \cdot 25$ & 53.93 & $54 \cdot 70$ & 54.07 & 54.58 & 54.89 & $56 \cdot 20$ & $56 \cdot 06$ & $52 \cdot 97$ & $53 \cdot 34$ \\
\hline $\mathrm{TiO}_{2}$ & 0.23 & 0.24 & 0.34 & 0.18 & 0.21 & 0.30 & 0.16 & 0.26 & 0.10 & 0.19 \\
\hline $\mathrm{Al}_{2} \mathrm{O}_{3}$ & $5 \cdot 65$ & $4 \cdot 11$ & $2 \cdot 98$ & $5 \cdot 67$ & 4.53 & $3 \cdot 44$ & $2 \cdot 39$ & $2 \cdot 85$ & $5 \cdot 13$ & $6-04$ \\
\hline $\mathrm{Cr}_{2} \mathrm{O}_{3}$ & 0.55 & 0.66 & 0.61 & 0.70 & 0.65 & 0.61 & 0.54 & 0.63 & 0.64 & 0.50 \\
\hline $\mathrm{FeO}$ & $6 \cdot 67$ & $6 \cdot 75$ & 7.02 & 6.49 & 6.96 & $6 \cdot 51$ & $6 \cdot 99$ & $6 \cdot 66$ & 6.91 & $6 \cdot 37$ \\
\hline MnO & 0.13 & 0.14 & 0.13 & 0.14 & $0 \cdot 18$ & 0.13 & 0.18 & 0.13 & 0.20 & 0.16 \\
\hline $\mathrm{MgO}$ & 32.98 & 33.49 & 33.93 & $31 \cdot 67$ & $32 \cdot 84$ & 33.02 & $33 \cdot 60$ & $33 \cdot 41$ & 33.95 & $32 \cdot 58$ \\
\hline $\mathrm{CaO}$ & 0.77 & 0.79 & 0.81 & 1.87 & 0.79 & $0-83$ & 0.52 & 0.89 & 0.76 & 0.93 \\
\hline $\mathrm{Na}_{2} \mathrm{O}$ & 0.03 & 0.00 & 0.00 & 0.09 & 0.00 & 0.00 & 0.00 & 0.03 & 0.09 & 0.00 \\
\hline $\mathrm{K}_{2} \mathrm{O}$ & 0.00 & 0.00 & 0.00 & 0.00 & 0.00 & 0.00 & 0.00 & 0.00 & 0.00 & 0.00 \\
\hline \multirow[t]{3}{*}{ Total } & $100 \cdot 26$ & $100 \cdot 11$ & $100 \cdot 53$ & $100 \cdot 88$ & $100 \cdot 74$ & $99 \cdot 73$ & $100 \cdot 58$ & $100 \cdot 92$ & $100 \cdot 76$ & $100 \cdot 11$ \\
\hline & \multicolumn{2}{|c|}{ ER-N2/1 } & \multicolumn{3}{|c|}{ ER-N2/18 } & ER-N2/16 & \multicolumn{2}{|l|}{ ER-R3/3 } & \multicolumn{2}{|l|}{ ER-S2/2 } \\
\hline & $p / 1 / r$ & $\mathrm{~g} / 2$ & $p / 1 / c$ & $p / 1 / r$ & $g / 2$ & $p / 1 / c$ & $p / 1 / c^{*}$ & $p / 1 / r^{*}$ & $p / 1 / c^{*}$ & $g / 2^{\circ}$ \\
\hline $\mathrm{SiO}_{2}$ & $54 \cdot 46$ & 54.89 & $53 \cdot 88$ & 54.09 & 54.99 & $54 \cdot 50$ & $53 \cdot 81$ & $53 \cdot 35$ & $56 \cdot 04$ & $55 \cdot 59$ \\
\hline $\mathrm{TiO}_{2}$ & 0.19 & 0.26 & 0.17 & 0.20 & 0.26 & 0.04 & 0.18 & 0.18 & 0.29 & 0.15 \\
\hline $\mathrm{Al}_{2} \mathrm{O}_{3}$ & 4.51 & 3.51 & 5.63 & 5.02 & $2 \cdot 78$ & 5.54 & $6 \cdot 11$ & $5 \cdot 88$ & 6.45 & $2 \cdot 33$ \\
\hline $\mathrm{Cr}_{2} \mathrm{O}_{3}$ & 0.55 & 0.54 & 0.54 & 0.61 & 0.53 & 0.51 & 0.32 & 0.38 & 0.55 & 0.20 \\
\hline $\mathrm{FeO}$ & 6.39 & 6.65 & 6.82 & 6.73 & 6.77 & $7 \cdot 17$ & $6 \cdot 31$ & $6 \cdot 22$ & $5 \cdot 37$ & $7 \cdot 23$ \\
\hline $\mathrm{MnO}$ & 0.13 & 0.16 & 0.17 & $0 \cdot 16$ & 0.15 & 0.22 & 0.08 & 0.15 & 0.09 & 0.16 \\
\hline $\mathrm{MgO}$ & 33.51 & $33 \cdot 31$ & $32 \cdot 18$ & 32.47 & 33.45 & $31 \cdot 86$ & $32 \cdot 28$ & $33 \cdot 05$ & 30.52 & $34 \cdot 48$ \\
\hline $\mathrm{CaO}$ & 0.75 & 0.87 & 0.70 & 0.78 & 0.82 & 0.59 & 0.87 & 0.68 & 1.00 & 0.36 \\
\hline $\mathrm{Na}_{2} \mathrm{O}$ & 0.04 & 0.03 & 0.00 & 0.02 & 0.04 & 0.17 & 0.07 & 0.06 & 0.38 & 0.00 \\
\hline $\mathrm{K}_{2} \mathrm{O}$ & 0.01 & 0.00 & 0.00 & 0.00 & 0.00 & 0.00 & 0.00 & 0.00 & 0.00 & 0.00 \\
\hline Total & 100.54 & $100 \cdot 22$ & 100.09 & 100.08 & $39 \cdot 79$ & $100 \cdot 60$ & $100 \cdot 03$ & 99.95 & $100-69$ & $100 \cdot 50$ \\
\hline
\end{tabular}

p, porphyroclast; g, granoblast; c, core; r, rim; ex, exsolution in clinopyroxene porphyroclast.

"Data from Rampone et al. (1993).

Table 7: Major element compositions of plagioclases

\begin{tabular}{|c|c|c|c|c|c|c|c|c|c|c|c|c|c|c|c|}
\hline & \multirow{2}{*}{$\frac{E R-N 1 / 4}{r / 1}$} & \multirow{2}{*}{$\begin{array}{l}\text { ER-N1/3 } \\
g / 1^{*}\end{array}$} & \multicolumn{2}{|c|}{ ER-N1/5 } & \multirow{2}{*}{$\frac{\mathrm{ER}-\mathrm{N} 1 / 2}{r / 1^{*}}$} & \multicolumn{2}{|c|}{ ER-N2/1 } & \multicolumn{2}{|c|}{ ER-N2/18 } & \multicolumn{2}{|c|}{ ER-N2/16 } & \multicolumn{2}{|l|}{ ER-R3/3 } & \multicolumn{2}{|l|}{ ER-S2/2 } \\
\hline & & & $g / 1$ & $e x / 2$ & & $r / 1$ & $g / 2$ & $e x / 1$ & $g / 2$ & $r / 1$ & $g / 2$ & $r / 1$ & $g / 2^{*}$ & $r / 1^{\circ}$ & $g / 2^{*}$ \\
\hline $\mathrm{SiO}_{2}$ & 52.52 & 53.32 & 51.90 & 51.41 & 50.05 & 51.63 & 51.98 & 52.82 & 52.89 & 51.69 & 51.41 & $54 \cdot 85$ & 53.54 & 52.51 & $52 \cdot 94$ \\
\hline $\mathrm{Al}_{2} \mathrm{O}_{3}$ & $30 \cdot 38$ & $29 \cdot 25$ & 30.62 & $31 \cdot 17$ & 31.91 & 31.85 & $31 \cdot 17$ & $30 \cdot 38$ & $30 \cdot 23$ & 30.93 & $31 \cdot 28$ & $29 \cdot 30$ & $28 \cdot 17$ & $31 \cdot 12$ & 29.99 \\
\hline $\mathrm{FeO}$ & 0.16 & 0.15 & 0.13 & 0.14 & 0.20 & 0.16 & 0.18 & 0.17 & 0.15 & 0.09 & 0.31 & 0.13 & 0.16 & 0.00 & 0.31 \\
\hline $\mathrm{CaO}$ & $12 \cdot 80$ & 12.07 & $13 \cdot 24$ & 13.57 & 14.80 & $11 \cdot 28$ & 13.52 & $12 \cdot 60$ & $12 \cdot 76$ & 12.56 & 13.78 & $10 \cdot 72$ & 13.48 & 12.44 & $12 \cdot 04$ \\
\hline $\mathrm{Na}_{2} \mathrm{O}$ & $4 \cdot 38$ & $4 \cdot 88$ & $4 \cdot 14$ & $3 \cdot 80$ & $3 \cdot 22$ & 4.94 & 4.03 & 4.69 & $4 \cdot 50$ & 4.40 & $3 \cdot 80$ & 5.55 & $4 \cdot 96$ & 4.03 & 4.86 \\
\hline $\mathrm{K}_{2} \mathrm{O}$ & 0.01 & 0.02 & 0.02 & 0.01 & 0.00 & 0.12 & 0.01 & 0.02 & 0.02 & 0.01 & 0.02 & 0.08 & 0.10 & 0.00 & 0.08 \\
\hline Total & 100.25 & 99.69 & 100.05 & $100 \cdot 10$ & $100 \cdot 18$ & 99.98 & $100 \cdot 89$ & $100 \cdot 68$ & 100.55 & 99.68 & $100 \cdot 60$ & 100.63 & 100.41 & $100 \cdot 10$ & $100 \cdot 22$ \\
\hline
\end{tabular}

r, rim around spinel; g, grain in pl + ol + px granoblastic domains; ex, exsolution in clinopyroxene.

- Data from Rampone et al. (1993). 
Table 8: Major element compositions of amphiboles

\begin{tabular}{|c|c|c|c|c|c|c|c|c|c|c|c|}
\hline & \multicolumn{2}{|c|}{ ER-N1/4 } & \multirow{2}{*}{$\begin{array}{l}\mathrm{ER}-\mathrm{N} 1 / 5 \\
\mathrm{~g} / 1\end{array}$} & \multicolumn{2}{|c|}{ ER-N2/1 } & \multicolumn{2}{|c|}{ ER-N2/18 } & \multicolumn{3}{|c|}{ ER-R3/3 } & \multirow{2}{*}{$\begin{array}{l}\text { ER-S2/2 } \\
\mathrm{g} / 1^{*}\end{array}$} \\
\hline & $g / 1^{\circ}$ & $g / 2$ & & $g / 1$ & $g / 2$ & $g / 1$ & $\mathrm{~g} / 2$ & $p / 1 / c$ & $p / 1 / r$ & $g / 2$ & \\
\hline $\mathrm{SiO}_{2}$ & $43 \cdot 16$ & 43.02 & 42.03 & 42.92 & $42 \cdot 34$ & $43 \cdot 71$ & $43 \cdot 41$ & $42 \cdot 24$ & $42 \cdot 48$ & $42 \cdot 38$ & $43 \cdot 29$ \\
\hline $\mathrm{TiO}_{2}$ & $3 \cdot 36$ & 3.39 & 3.69 & $4 \cdot 28$ & $4 \cdot 34$ & $3 \cdot 12$ & 3.28 & 5.63 & 5.46 & 3.68 & $3 \cdot 20$ \\
\hline $\mathrm{Al}_{2} \mathrm{O}_{3}$ & $12 \cdot 86$ & 12.95 & 12.75 & $12 \cdot 62$ & $12 \cdot 63$ & $12 \cdot 72$ & $12 \cdot 87$ & 13.08 & 13.90 & $14 \cdot 85$ & 13.59 \\
\hline $\mathrm{Cr}_{2} \mathrm{O}_{3}$ & 1.29 & 1.47 & 1.60 & 1.51 & 1.53 & $1 \cdot 72$ & 1.64 & $1 \cdot 39$ & $1 \cdot 22$ & 0.89 & $1 \cdot 27$ \\
\hline $\mathrm{FeO}$ & 4.59 & $4 \cdot 36$ & $5 \cdot 40$ & 4.46 & $4 \cdot 94$ & $4 \cdot 33$ & $4 \cdot 30$ & 4.06 & $4 \cdot 10$ & 3.91 & $4 \cdot 29$ \\
\hline Mno & 0.05 & 0.08 & 0.09 & 0.10 & 0.06 & 0.05 & 0.11 & 0.07 & 0.08 & 0.04 & 0.08 \\
\hline MgO & $18 \cdot 11$ & $17 \cdot 55$ & $17 \cdot 26$ & $17 \cdot 17$ & $17 \cdot 76$ & 17.79 & $17 \cdot 50$ & 16.40 & 16.51 & $17 \cdot 28$ & $17 \cdot 28$ \\
\hline $\mathrm{CaO}$ & $12 \cdot 20$ & 11.99 & $11 \cdot 17$ & $11 \cdot 18$ & 11.72 & 12.03 & 12.04 & $11 \cdot 76$ & $11 \cdot 87$ & 11.69 & $12 \cdot 23$ \\
\hline $\mathrm{Na}_{2} \mathrm{O}$ & 3.00 & $3 \cdot 24$ & 3.02 & $3 \cdot 20$ & $3 \cdot 26$ & $3 \cdot 31$ & 3.44 & 3.47 & 3.39 & $3 \cdot 28$ & 3.39 \\
\hline $\mathrm{K}_{2} \mathrm{O}$ & 0.04 & 0.01 & 0.04 & 0.13 & 0.11 & 0.06 & 0.01 & 0.41 & 0.44 & 0.64 & 0.33 \\
\hline Total & $98 \cdot 66$ & 98.06 & 97.95 & $97 \cdot 57$ & 98.69 & $98 \cdot 84$ & $98 \cdot 60$ & 98.51 & 98.56 & $98 \cdot 64$ & 98.95 \\
\hline
\end{tabular}

p, porphyroclast; g, granoblast; c, core; r, rim.

-Data from Rampone et al. (1993).

Table 9: Trace element mineral compositions of Type A samples

\begin{tabular}{|c|c|c|c|c|c|c|c|c|c|c|c|c|c|}
\hline \multirow[b]{4}{*}{ Ce } & \multicolumn{9}{|l|}{ ER-R3/3 } & \multicolumn{4}{|l|}{ ER-S2/2 } \\
\hline & $\mathrm{cpx}{ }^{*}$ & cpx $x^{*}$ & $\operatorname{cpx} x^{*}$ & $c p x^{\circ}$ & cpx ${ }^{*}$ & $o p x^{*}$ & amph & amph & amph & $\operatorname{cpx}{ }^{\circ}$ & $\operatorname{cpx} x^{*}$ & $\operatorname{cpx}{ }^{*}$ & opx \\
\hline & $p / 1 / c$ & $g / 2$ & $p / 3 / c$ & $p / 3 / r$ & $p / 4 / c$ & $p / 5$ & $p / 6 / c$ & $\mathrm{p} / 7$ & $g / 9$ & $p / 1 / c$ & $p / 1 / r$ & $g / 2$ & $p / 3 / c$ \\
\hline & $5 \cdot 69$ & 7.65 & $6 \cdot 71$ & 6.65 & 5.07 & 0.020 & $6 \cdot 10$ & $4 \cdot 58$ & $6 \cdot 55$ & 6.43 & $6 \cdot 89$ & $5 \cdot 80$ & 0.024 \\
\hline Nd & $5 \cdot 49$ & 8.05 & $6 \cdot 41$ & 6.42 & $4 \cdot 70$ & 0.049 & 6.51 & $4 \cdot 67$ & 7.51 & $5 \cdot 85$ & $6 \cdot 88$ & 6.68 & 0.052 \\
\hline Sm & 1.75 & 2.91 & 2-32 & $2 \cdot 24$ & 1.69 & 0.025 & $2 \cdot 64$ & 1.90 & $3 \cdot 21$ & $2 \cdot 19$ & $2 \cdot 78$ & $2 \cdot 31$ & 0.030 \\
\hline Eu & 0.83 & 1.23 & $1 \cdot 13$ & 1.07 & 0.78 & 0.014 & 1.17 & 0.90 & 1.30 & 1.03 & 1.10 & 0.96 & 0.014 \\
\hline Gd & - & - & 3.38 & 3.44 & 2.62 & - & 3.49 & $2 \cdot 70$ & $4 \cdot 37$ & $2 \cdot 93$ & 3.97 & 3.33 & 0.075 \\
\hline Dy & $2 \cdot 68$ & $4 \cdot 73$ & 3.99 & 3.85 & $3 \cdot 15$ & 0.09 & $4 \cdot 33$ & $3 \cdot 47$ & $5 \cdot 91$ & 3.67 & 4.76 & 3.66 & 0.19 \\
\hline Er & 1.56 & $2 \cdot 63$ & 2.46 & $2 \cdot 41$ & 1.89 & 0.10 & $2 \cdot 86$ & $2 \cdot 36$ & 4.09 & $2 \cdot 24$ & 3.02 & $2 \cdot 31$ & 0.25 \\
\hline Yb & 1.49 & $2 \cdot 42$ & $2 \cdot 30$ & $2 \cdot 23$ & 1.66 & 0.12 & $2 \cdot 90$ & $2 \cdot 42$ & 4.05 & $2 \cdot 07$ & $2 \cdot 74$ & $2 \cdot 20$ & 0.31 \\
\hline $\mathrm{Na}$ & 11946 & 5758 & 12617 & 12172 & 11601 & 563 & - & - & - & 5489 & 4410 & 5362 & 274 \\
\hline Sc & 62 & 89 & 56 & 56 & 52 & 12 & - & - & - & 63 & 80 & 68 & $21 \cdot 6$ \\
\hline $\mathrm{Ti}$ & 3560 & 5729 & 3735 & 3801 & 3603 & 725 & 16601 & 20360 & 28149 & 3407 & 4809 & 3351 & 773 \\
\hline$v$ & 222 & 281 & 216 & 219 & 210 & 88 & 510 & 608 & 644 & 227 & 278 & 247 & 128 \\
\hline $\mathrm{Cr}$ & 4591 & 3775 & 3415 & 3418 & 4409 & 2057 & - & - & - & 4599 & 4494 & 5165 & 2347 \\
\hline Sr & 58 & 11 & 66 & 61 & 61 & 0.4 & 196 & 132 & 172 & 20 & $7 \cdot 1$ & 14 & 0.18 \\
\hline $\mathrm{z}$ & 52 & 91 & 45 & 45 & 45 & 2.5 & 40 & 42 & 55 & 46 & 79 & 46 & 3.87 \\
\hline$Y$ & 21 & 33 & 22 & 22 & 19 & $1 \cdot 2$ & 33 & 42 & 32 & 24 & 34 & 23 & 2.00 \\
\hline $\mathrm{Ba}$ & - & - & - & - & - & - & $8 \cdot 6$ & $12 \cdot 6$ & 20 & - & - & - & - \\
\hline$K$ & - & - & - & - & - & - & 3165 & 4240 & 2688 & - & - & - & - \\
\hline
\end{tabular}

p, porphyroclast; g, granoblast; c, core; r, rim.

-Data from Rampone et al. (1993). 


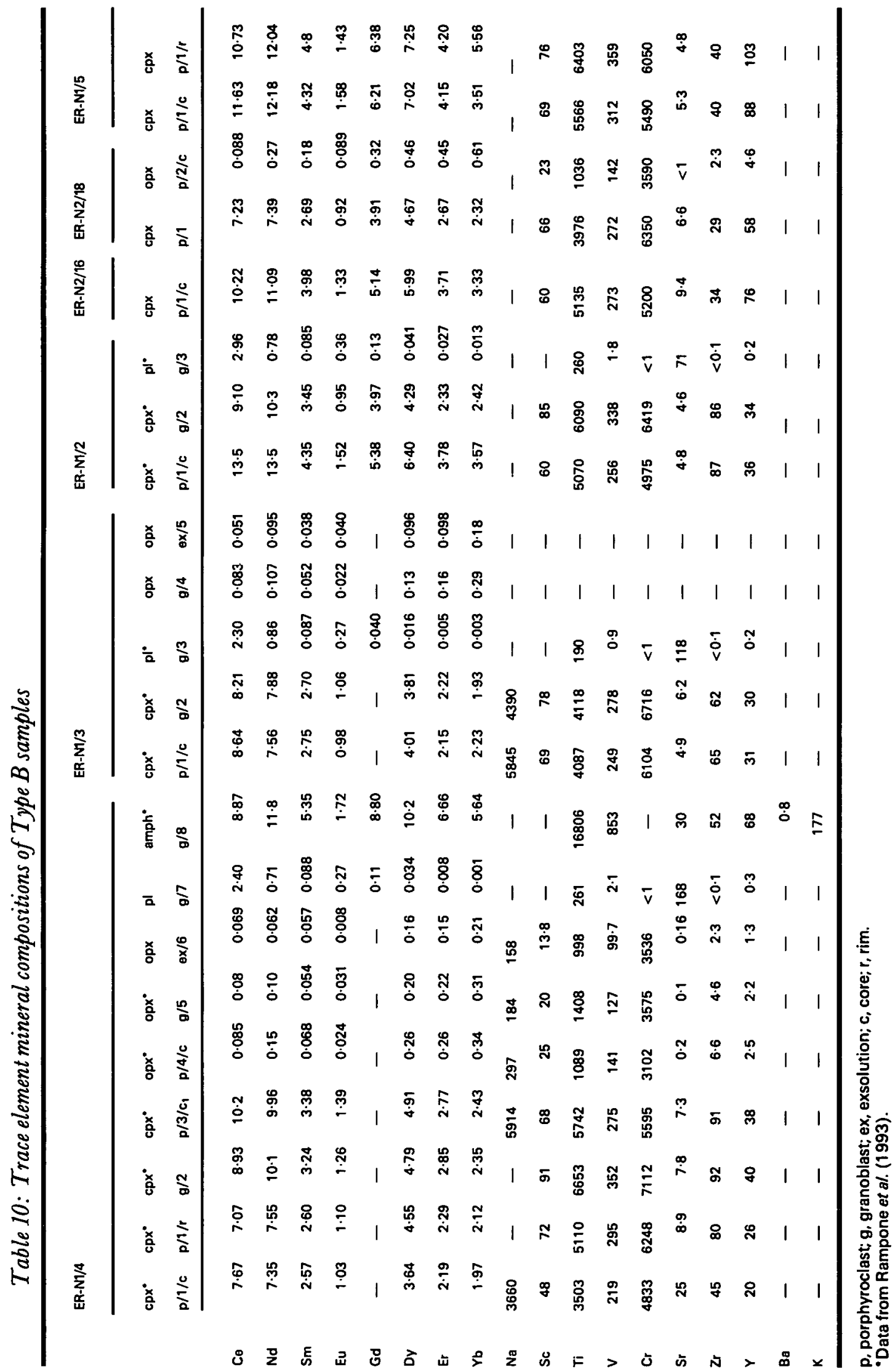




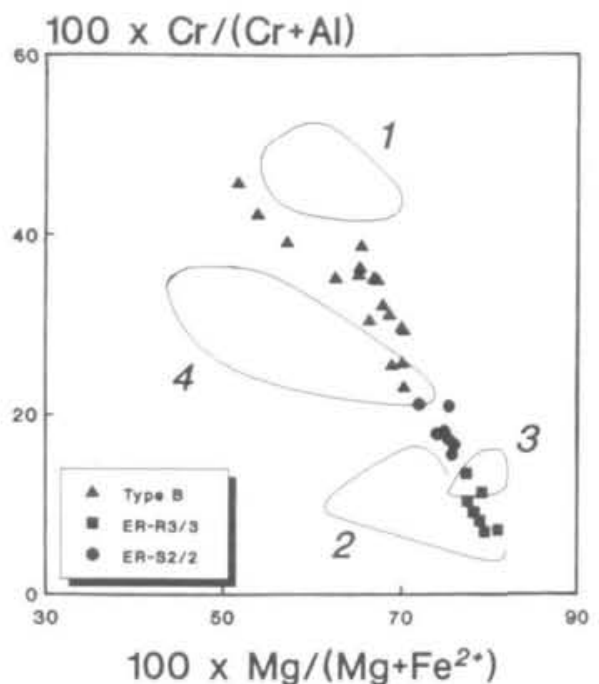

Fig. 5. $\mathrm{Cr} /(\mathrm{Cr}+\mathrm{Al})(\times 100)$ vs $\mathrm{Mg} /\left(\mathrm{Mg}^{2}+\mathrm{Fe}^{2+}\right)(\times 100)$ (atoms per four oxygens) for spinels from the EL peridotites. The fields refer to the composition of spinels in peridotites from: (1) Internal Ligurides (IL), Northern Apennines (Rampone, 1992), (2) Baldissero and Balmuccia (less depleted), Ivrea Zone (Rivalenti et al., 1979), (3) and (4) Erro-Tobbio, Ligurian Alps (spinel lherzolites and plagioclase lherzolites, respectively) (Piccardo et al., 1990; Hoogerduijn Strating et al., 1993).

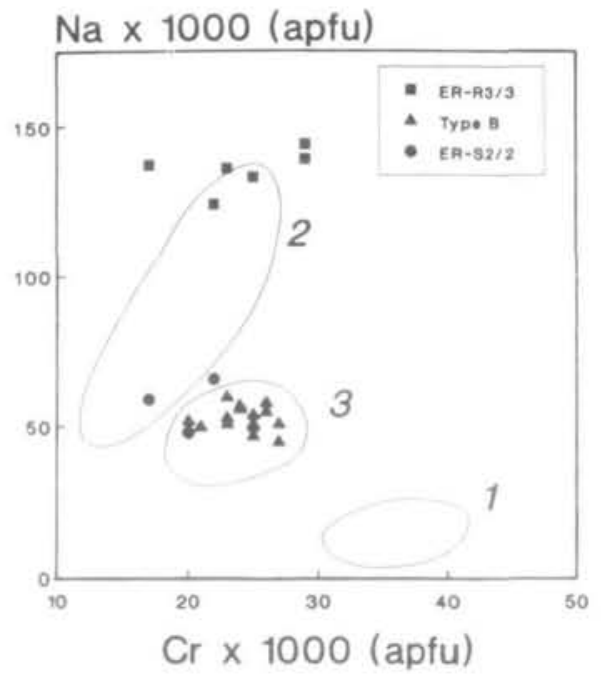

Fig. 6. Variation of $\mathrm{Na}(\times 1000)$ vs $\mathrm{Cr}(\times 1000)$ (atoms per six oxygens) for spinel-facies clinopyroxene porphyroclasts from the EL peridotites. Fields as in Fig. 5. Apfu, atoms per formula unit.

clinopyroxenes in Type B samples are generally characterized by significantly lower $\mathrm{Sr}$ contents ( $<10$ p.p.m.), coupled with higher $\mathrm{Zr}, \mathrm{Y}, \mathrm{Ti}, \mathrm{V}$ and Sc concentrations (Fig. 8a). Mass balance calculations (Rampone et al., 1993) have demonstrated that the observed variations in the clinopyroxene trace element composition are the result of the element redistribution related to subsolidus re-equilibration from spinel- to plagioclase-facies conditions.

\section{Orthopyroxene}

Orthopyroxenes are enstatites, with $\mathrm{Mg}$ / $\left(\mathrm{Mg}+\mathrm{Fe}^{2+}\right)$ in the range of $0.892-0.907$. Spinelfacies orthopyroxenes exhibit high $\mathrm{Al}_{2} \mathrm{O}_{3}$ (up to 6 wt $\%$ ) and $\mathrm{CaO}$ (up to 1 wt \%) contents. Both $\mathrm{Al}$ and $\mathrm{Ca}$ decrease in plagioclase-facies granoblastic orthopyroxenes.

Orthopyroxenes are characterized by very low REE absolute concentrations $[<(1-2) \times \mathrm{C} 1]$ and strongly fractionated patterns $\left(\mathrm{Ce}_{\mathcal{N}} / \mathrm{Yb}_{\mathcal{N}} 0.02-0.07\right)$ (Fig. 7b). This is in agreement with literature data for REE compositions of orthopyroxene in mantle peridotites (Stosch \& Seck, 1980; Stosch, 1982; Rampone et al., 1991). The orthopyroxenes also show very low contents of $\mathrm{Sr}(<1$ p.p.m.) and $\mathrm{Y}$ (1.5-3 p.p.m.), whereas $\mathrm{Zr}$ ranges from 2 to 7 p.p.m. (Fig. 8b). These values are consistent with recently published trace element data for orthopyroxene in sp lherzolite xenoliths (O'Reilly et al., 1991). Larger variations for $\mathrm{Ti}$ (700-1400 p.p.m.), V (88-140 p.p.m.) and Sc (12-25 p.p.m.) (Fig. 8b) can be related to subsolidus effects (Rampone et al., 1993).

\section{Amphibole}

According to Leake's (1978) classification, amphiboles range from kaersutite to Ti-rich pargasite (and subordinate $\mathrm{Mg}$-hastingsite) types. Kaersutitic amphiboles $\left(\mathrm{TiO}_{2}\right.$ up to 5-6 wt \%) occur only in sample ER-R3/3; they are part of the spinel-facies paragenesis. Amphiboles recrystallized in equilibrium with plagioclase have $\mathrm{Ti}$-pargasitic compositions $\left(\mathrm{TiO}_{2}\right.$ in the range 2.96-3.97 wt \%). A similar compositional trend, namely a $\mathrm{Ti}$ decrease from spto pl-facies amphiboles, has also been documented for the pre-oceanic rift lherzolites of Zabargad (Red Sea) (Piccardo et al., 1988). All the analysed amphiboles have very low $\mathrm{K}$ contents $\left(\mathrm{K}_{2} \mathrm{O}<0.4 \mathrm{wt} \%\right)$.

The REE spectra are similar to those of coexisting clinopyroxenes (Fig. 7b). They show slight LREE depletion $\left(\mathrm{Ce}_{\mathcal{N}} / \mathrm{Sm}_{\mathcal{N}}=0.5-0.6\right)$ and almost flat M- to HREE patterns at 16-22 times C1. Comparable REE abundances have been documented for kaersutite-Ti-pargasite amphiboles in the Zabargad sp lherzolites (Piccardo et al., 1993) and in sp lherzolite xenoliths from the Baikal rift zone (Ionov et al., 1992) and the Arabian side of the Red Sea (HenjesKunst et al., 1990). REE patterns of sp-facies amphiboles are generally similar to the respective clinopyroxene patterns. They display lower REE absolute concentrations $[(10-18) \times \mathrm{C} 1]$ and positive $\mathrm{Eu}_{\mathcal{N}}$ anomalies (Fig. $7 \mathrm{~b}$ ), whereas pl-facies amphiboles have higher REE abundances [up to (30$40) \times \mathrm{Cl}$ ] and develop negative $\mathrm{Eu}_{\mathcal{N}}$ anomalies (Eu/ $\mathrm{Eu}^{*}=0 \cdot 76-0 \cdot 82$ ). 

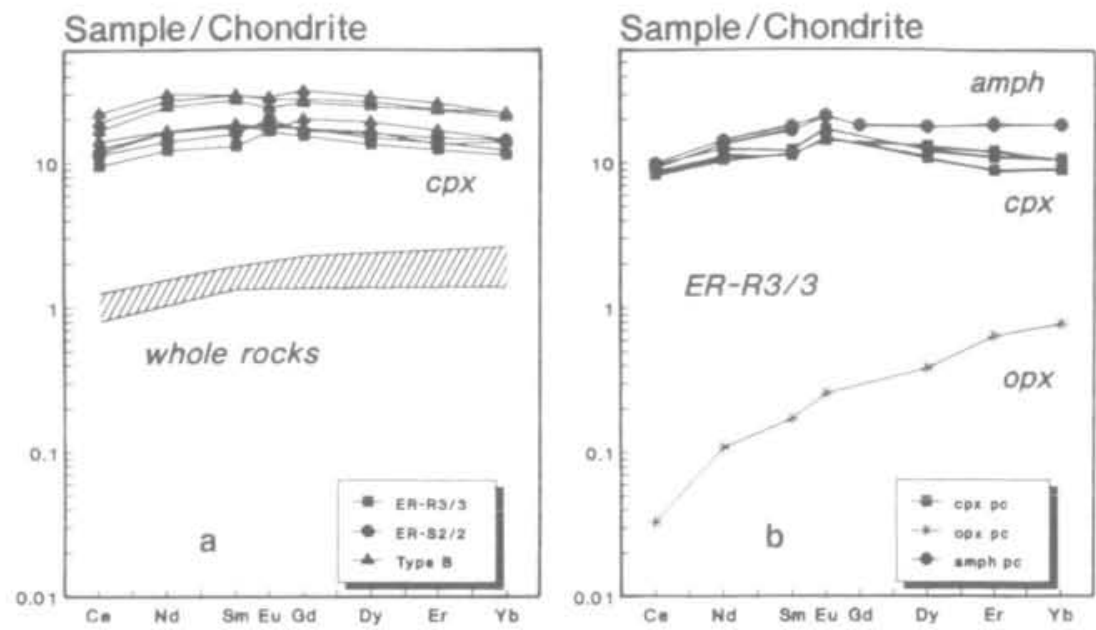

Fig. 7. (a) Chondrite-normalized REE abundances for sp-facies porphyroclastic clinopyroxenes from sp lherzolites (Type A, samples ER-R3/3 and ER-S2/2) and as relics in pl lherzolites (Type B) (averages of analyses listed in Tables 9 and 10). Normalizing values are taken from Anders \& Ebihara (1982). The dashed field refers to bulk-rock REE compositions of EL peridotites available in literature (Beccaluva et al., 1984; Ottonello et al., 1984). (b) Chondrite-normalized REE representative concentrations for orthopyroxene (opx), clinopyroxene (cpx) and amphibole (amph) pertaining to the sp-facies assemblage in sample ER-R3/3. pc, porphyroclast core.
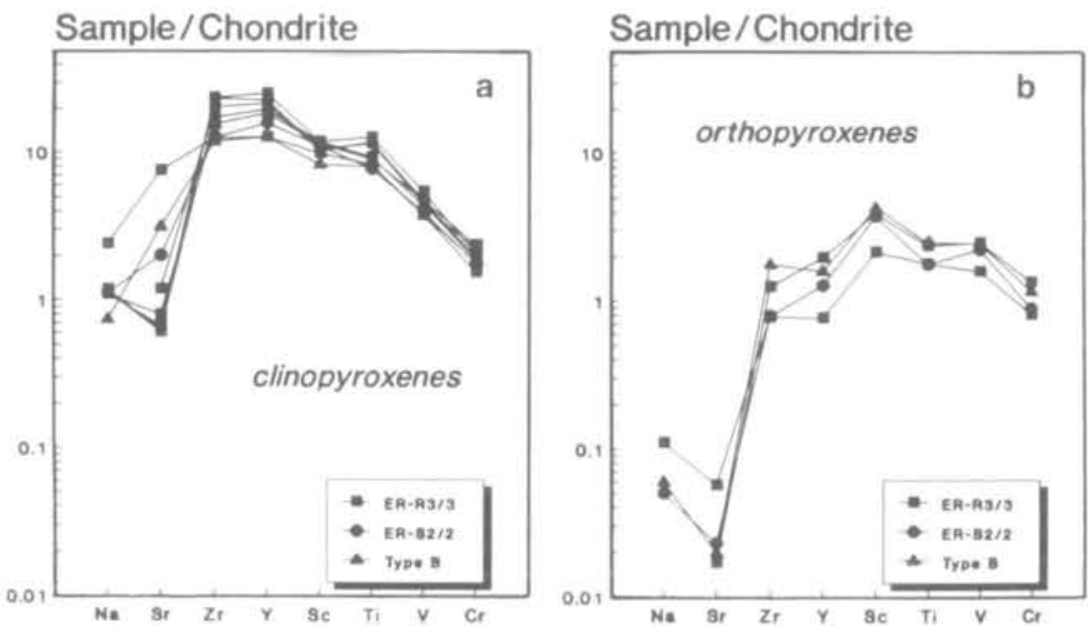

Fig. 8. Chondrite-normalized trace element $(\mathrm{Na}, \mathrm{Sr}, \mathrm{Zr}, \mathrm{Y}, \mathrm{Sc}, \mathrm{Ti}, \mathrm{V}$ and $\mathrm{Cr}$ ) representative concentrations in sp-facies clinopyroxenes (a) and orthopyroxenes (b) from the EL peridotites (averages of analyses listed in Tables 9 and 10). Symbols as in Fig. 5.

EL amphiboles show, moreover, low $\mathrm{Sr}(<200$ p.p.m.) and $\mathrm{Ba}$ ( $<20$ p.p.m.) contents relative to amphiboles crystallized from alkaline melts (Basu, 1978; Menzies \& Hawksworth, 1987). Similarly low $\mathrm{Sr}$ and $\mathrm{Ba}$ concentrations have been documented in sp-facies amphiboles from the Zabargad lherzolites (Piccardo et al., 1993) (Fig. 9).

\section{Plagioclase}

Plagioclase in the EL lherzolites is a subsolidus reaction product. Its compositional features and the relationships with the chemical variations in coexisting minerals have been discussed by Beccaluva et al. (1984) and Rampone et al. (1993).

Plagioclases range from $A n_{45}$ to $A n_{70}$. No correlations have been observed between An composition and textural occurrence (granoblastic grains, exsolution in $\mathrm{cpx}$, rims around spinel). Their REE patterns are strongly fractionated, with significant LREE enrichment $\left(\mathrm{Ce}_{\mathcal{N}} / \mathrm{Yb}_{\mathcal{N}}=26-62, \quad \mathrm{Sm}_{\mathcal{N}}\right)$ $\left.\mathrm{Nd}_{\mathcal{N}}=0.11-0.44\right)$, well-developed positive $\mathrm{Eu}$ anomalies $\left[\mathrm{Eu}_{\mathcal{N}}=(4 \cdot 3-5 \cdot 7) \times \mathrm{Cl}\right]$ and very low HREE abundances $(<0.2 \times \mathrm{Cl})$, as usually reported in the literature (Grauch, 1989; McKay, 1989). EL plagicclases exhibit, moreover, high $\mathrm{Sr}$ contents 
Ba p.p.m.

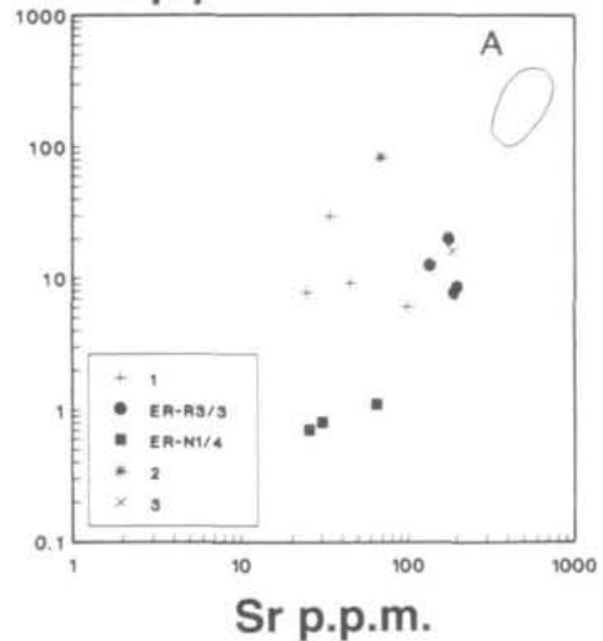

Fig. 9. Variation of $\mathrm{Ba}$ vs $\mathrm{Sr}$ concentrations (in p.p.m.) for amphiboles from ER-R3/3 and ER-N1/4 samples. For comparison, we have reported representative compositions of amphiboles in sp lherzolites from: (1) Zabargad (Red Sea), (2) Fontete Rouge and (3) Lherz (Eastern Pyrenees) [data from Piccardo et al. (1993) and author's unpublished data]. Field A refers to the compositions of kaersutite megacrysts in alkaline lavas (Basu, 1978).

(100-200 p.p.m.), and negligible $\mathrm{V}, \mathrm{Y}$ and $\mathrm{Zr}$ abundances ( $<1$ p.p.m.).

\section{Sr AND Nd ISOTOPE GEOCHEMISTRY}

$\mathrm{Sr}$ and $\mathrm{Nd}$ isotopic compositions of separated clinopyroxenes are listed in Table 11. Most samples display a limited range $\left({ }^{87} \mathrm{Sr} /{ }^{86} \mathrm{Sr}=0 \cdot 702216-0 \cdot 702634\right.$; $\left.{ }^{43} \mathrm{Nd} /{ }^{144} \mathrm{Nd}=0.513047-0.513205\right)$, and plot within the depleted end of the MORB field (White, 1985) (Fig. 10). Sm and Nd concentrations obtained by isotope dilution (Table 11) are consistent with the ion probe data. ${ }^{147} \mathrm{Sm} /{ }^{144} \mathrm{Nd}$ ratios range from 0.232 to $0 \cdot 253$, and are consistent with ${ }^{147} \mathrm{Sm} /{ }^{144} \mathrm{Nd}$ ratio $(0.222-0.25)$ characteristic of the depleted mantle reservoir (Faure, 1986; Zindler \& Hart, 1986).

Sample ER-S2/2 is peculiar, being characterized by extremely depleted isotopic composition $\left({ }^{87} \mathrm{Sr} /{ }^{86} \mathrm{Sr}=0.701736 ;{ }^{143} \mathrm{Nd} /{ }^{144} \mathrm{Nd}=0.513543\right)$, so that it plots on an extension of the oceanic mantle array towards residual mantle composition (Fig. 10). Such a low value is unusual for Phanerozoic mantle rocks, and bears some similarity to values of peridotite xenoliths from Proterozoic continental lithosphere (Stosch et al., 1986; Stosch \& Lugmair, 1986; Menzies \& Hawkesworth, 1987; Roden et al., 1988; Galer \& O'Nions, 1989).

In Fig. 10, $\mathrm{Sr}$ and $\mathrm{Nd}$ isotopic compositions of EL peridotites are compared with those of orogenic peridotites from the Western Alps [e.g. Lanzo and Baldissero; data from Bodinier et al. (1991) and Obermiller et al. (unpub. data, 1994)]. For the EL samples, the measured values have been plotted directly. No age correction is needed for the $\mathrm{Sr}$ isotope ratios, because of the very low $\mathrm{Rb} / \mathrm{Sr}$ ratios in mantle clinopyroxene. The ${ }^{143} \mathrm{Nd} /{ }^{144} \mathrm{Nd}$ ratios were also left uncorrected, because the ${ }^{147} \mathrm{Sm} /{ }^{144} \mathrm{Nd}$ ratios of analysed samples are very similar to unmodified MORB mantle. However, a decay correction was necessary for the Lanzo and Baldissero peridotites, to make the data comparable with present-day MORB mantle and with the EL lherzolites, because most of ${ }^{147} \mathrm{Sm} /{ }^{144} \mathrm{Nd}$ ratios in Lanzo and Baldissero peridotites are higher than $0 \cdot 25$. The initial Nd isotopic compositions have been calculated assuming ages of emplacement of $270 \mathrm{Ma}$ for Baldissero and $200 \mathrm{Ma}$ for Lanzo (Voshage et al., 1988; Bodinier et al., 1991). The zero-age isotopic ratios, without the effect of depletion events, have been computed using ${ }^{147} \mathrm{Sm} /{ }^{144} \mathrm{Nd}=0.222$, characteristic of MORB mantle (Faure, 1986). The calculated ${ }^{143} \mathrm{Nd} /{ }^{144} \mathrm{Nd}$ ratios, coupled with measured ${ }^{87} \mathrm{Sr} /{ }^{86} \mathrm{Sr}$ compositions, have been used to draw the compositional fields reported in Fig. 10. It is noteworthy that the EL, Lanzo and Baldissero peridotites display very similar isotopic composition and are invariably characterized by MORB-type isotopic signatures. It must be emphasized that MORB isotopic compositions are typical for many subcontinental peridotites, both in orogenic peridotites (Polvé \& Allègre, 1980) and in spinel therzolite xenoliths from mobile beltrift valley regions (Jagoutz et al., 1980; Roden et al., 1984; Stosch \& Lugmair, 1986; Stosch et al., 1986; Menzies \& Hawkesworth, 1987). This is not surprising: presumably this similarity simply reflects a long-term depletion history of the upper mantle before the accretion of the continental lithosphere.

Noticeably, one sample from the Lanzo peridotite (Northern body) shows very depleted $\mathrm{Sr}$ isotopic composition, similar to ER-S2/2, and has been considered to represent subcontinental lithospheric mantle (Bodinier et al., 1991).

In Fig. 10, the compositional field for the IL peridotite is also reported. As for Lanzo and Baldissero peridotites, $\mathrm{Nd}$ isotopic ratios have been corrected back to the age of emplacement (150 Ma, based on palaeontologic ages on radiolarian cherts overlying the IL peridotites) (Marcucci \& Passerini, 1991). The zero-age Nd isotopic ratios have been calculated assuming ${ }^{147} \mathrm{Sm} /{ }^{144} \mathrm{Nd}=0.222$. In spite of the age correction, the IL peridotites exhibit extremely depleted Nd isotopic compositions, different from those of the EL peridotites. 
Table 11: $S r, \mathcal{N}$ d isotopic compositions and $S m, \mathcal{N d}$ concentrations for the EL peridotites

\begin{tabular}{|c|c|c|c|c|c|c|c|c|c|c|}
\hline Sample & Mineral & ${ }^{87} \mathrm{Sr} /{ }^{86} \mathrm{Sr}$ & ${ }^{143} \mathrm{Nd} /{ }^{144} \mathrm{Nd}$ & Sm (p.p.m.) & Nd (p.p.m.) & ${ }^{147} \mathrm{Sm} /{ }^{144} \mathrm{Nd}$ & $E_{N d}$ & $E_{N d}(t)$ & $\begin{array}{l}\text { Si model age } \\
\text { (CHUR) }\end{array}$ & $\begin{array}{l}\text { Nd model age } \\
\text { (CHUR) }\end{array}$ \\
\hline \multirow[t]{4}{*}{ ER-N2/16 } & $\operatorname{cpxd}$ & & $0.513103 \pm 18$ & $3 \cdot 297$ & $8 \cdot 21$ & 0.243 & $+9 \cdot 1$ & +8.09 & $1.99 \mathrm{Ga}$ & $1.55 \mathrm{Ga}$ \\
\hline & $\operatorname{cpxc}$ & $0.702216 \pm 24$ & $0.513116 \pm 17$ & & & & & & & \\
\hline & $\mathrm{plc}$ & $0.702227 \pm 17$ & & & & & & & & \\
\hline & pld & & $0.512944 \pm 16$ & 0.181 & $1 \cdot 145$ & 0.0955 & & & & \\
\hline ER-N2/18 & $\operatorname{cpxd}$ & $0.702316 \pm 13$ & $0.513205 \pm 11$ & 2.071 & 4.991 & 0.2509 & $+11 \cdot 1$ & +9.92 & $1.91 \mathrm{Ga}$ & $1.59 \mathrm{Ga}$ \\
\hline \multirow[t]{4}{*}{ ER-N1/3 } & $\operatorname{cpxd}$ & & $0.513145 \pm 7$ & $2 \cdot 212$ & $5 \cdot 626$ & 0.2377 & +9.9 & +9.03 & $1.77 \mathrm{Ga}$ & $1.88 \mathrm{Ga}$ \\
\hline & $c p \times c$ & $0.702488 \pm 19$ & & $2 \cdot 864$ & & & & & & \\
\hline & $\mathrm{plc}$ & $0.702481 \pm 10$ & & & & & & & & \\
\hline & pld & & $0.512996 \pm 10$ & 0.109 & 0.668 & 0.0984 & & & & \\
\hline \multirow[t]{3}{*}{ ER-S2/2 } & $\operatorname{cpx} d_{1}$ & & $0.513540 \pm 18$ & $2 \cdot 19$ & $5 \cdot 218$ & 0.254 & $+17 \cdot 6$ & $+15 \cdot 8$ & $2.36 \mathrm{Ga}$ & $2.45 \mathrm{Ga}$ \\
\hline & $\operatorname{cpxc}$ & $0.701750 \pm 36$ & $0.513544 \pm 21$ & $2 \cdot 348$ & $5 \cdot 622$ & 0.253 & $+17 \cdot 7$ & +16.5 & $1.2 \mathrm{Ga}^{*}$ & $2 \cdot 1 \mathrm{Gat}$ \\
\hline & $\operatorname{cpxd} d_{2}$ & $0.701722 \pm 22$ & $0.513546 \pm 15$ & $1 \cdot 838$ & $4-42$ & 0.252 & $+17 \cdot 7$ & +16.5 & & 779 Mał \\
\hline \multirow[t]{2}{*}{ ER-R3/3 } & $\operatorname{cpxd}$ & & $0.513169 \pm 17$ & $2 \cdot 072$ & $5 \cdot 262$ & 0.238 & $+10 \cdot 4$ & $+9 \cdot 5$ & $1.9 \mathrm{Ga}$ & $1.89 \mathrm{Ga}$ \\
\hline & $\operatorname{cpxc}$ & $0.702322 \pm 19$ & $0.513151 \pm 21$ & 1.967 & $4 \cdot 97$ & 0.239 & +10 & $+9 \cdot 1$ & & \\
\hline ER-N1/4 & $\operatorname{cpxd}$ & $0.702484 \pm 48$ & $0.513147 \pm 16$ & 2.668 & 6.611 & 0.244 & $+9 \cdot 9$ & $+8 \cdot 9$ & $1.7 \mathrm{Ga}$ & $1.64 \mathrm{Ga}$ \\
\hline ER-N $1 / 2$ & $\operatorname{cpxd}$ & $0.702634 \pm 25$ & $0.513047 \pm 17$ & $3 \cdot 207$ & $8 \cdot 379$ & 0.232 & +8 & $+7 \cdot 2$ & $1.66 \mathrm{Ga}$ & $1.79 \mathrm{Ga}$ \\
\hline ER-N1/5 & $\operatorname{cpxd}$ & $0.702519 \pm 28$ & $0.513074 \pm 16$ & $3 \cdot 106$ & 7.969 & 0.236 & +8.5 & $+7 \cdot 7$ & $1.75 \mathrm{Ga}$ & $1.70 \mathrm{Ga}$ \\
\hline
\end{tabular}

Sr model age: ${ }^{\circ} \mathrm{DM}$ model age $\left({ }^{87} \mathrm{Sr} /{ }^{86} \mathrm{Sr}_{\mathrm{OM}}=0.7026 ;{ }^{87} \mathrm{Rb} /{ }^{86} \mathrm{Sr} \mathrm{PM}_{2}=0.0509\right)$. Nd model age: $+\mathrm{DM} \mathrm{model}_{1} \mathrm{mge}$ $\left({ }^{143} \mathrm{Nd} / /^{144} \mathrm{Nd}_{\mathrm{DM}}=0.513114 ;{ }^{147} \mathrm{Sm} /{ }^{144} \mathrm{Nd}_{\mathrm{DM} 1}=0.222\right) ;\left\lceil D M_{2}\right.$ model age $\left({ }^{143} \mathrm{Nd} /{ }^{144} \mathrm{Nd} \mathrm{OM}_{2}=0.51335 ;{ }^{147} \mathrm{Sm} /{ }^{144} \mathrm{Nd} \mathrm{DM}_{2}=0.2148\right)$. $c$, 'clean' (carefully handpicked) separates; $d$, 'dirty' (direct output of the electromagnetic separator) separates (see the section on analytical procedures for explanation). $d_{1}$ and $d_{2}$ refer to different analysed aliquots in a single sample.

\section{P-T ESTIMATES}

The $P$ and $T$ nominal values for equilibration of EL peridotites under spinel- and plagioclase-facies conditions have been obtained using the composition of cpx-opx pairs pertaining to both assemblages. Temperatures have been calculated according to different geothermometers. The thermometers of Wood \& Banno (1973) and Wells (1977) are based on the exchange of $\mathrm{Ca}$ and $\mathrm{Mg}$ between clinopyroxene and orthopyroxene. The thermometer of Sachtleben \& Seck (1981) is based on the partitioning of $\mathrm{Al}$ between orthopyroxene and spinel, and is not applicable to plagioclase-bearing assemblages. Therefore, this method has been only applied to obtain the $T$ equilibration of the spinel-bearing assemblage. Mercier's (1980) thermobarometry furnishes $P$ and $T$ estimates from the composition of a singlepyroxene phase (in equilibrium with a second pyroxene and an $\mathrm{Al}$-rich mineral).
Application of the above methods yields mean equilibration temperatures which, for each assemblage, fall within a range of $100^{\circ} \mathrm{C}$. The thermometers of Wood \& Banno (1973) and Wells (1977) mark, respectively, the upper and lower bounds of this $T$ range.

The spinel-facies assemblage, based on compositions of homogeneous to slightly exsolved porphyroclast cores, yields mean temperatures between 1000 and $1050^{\circ} \mathrm{C}$, at $\sim 15-20$ kbar confining pressure. The plagioclase-facies re-equilibration, which has been determined using the compositions of recrystallized grains, yields lower-temperature conditions, mainly within the range $900-950^{\circ} \mathrm{C}$, and lower pressures $(8-10 \mathrm{kbar})$. The temperature estimates are consistent with equilibration conditions obtained by Beccaluva et al. (1984).

In spite of the controversial validity of barometers for spinel lherzolites, pressure estimates obtained by 


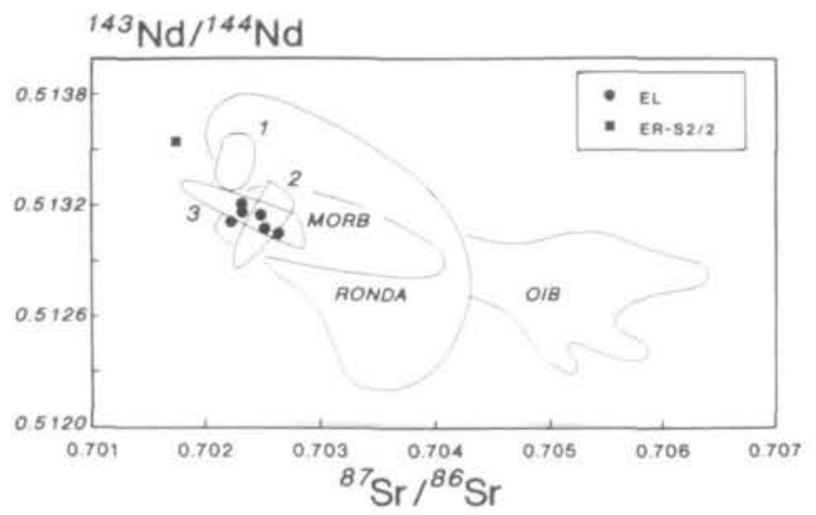

Fig. 10. Present-day ${ }^{143} \mathrm{Nd} /{ }^{144} \mathrm{Nd} \mathrm{vs}{ }^{87} \mathrm{Sr} /{ }^{86} \mathrm{Sr}$ diagram for clinopyroxenes separated from the EL peridotites. Also shown are the fields for MORBs and OIBs (Zindler \& Hart, 1986), as well as for clinopyroxenes from the lherzolite massifs of Ronda (Reisberg \& Zindler, 1987; Reisberg et al., 1989). Other fields refer to clinopyroxene data for peridotites from: (1) IL Units, Northern Apennine (Rampone, 1992), (2) Baldissero, Ivrea Zone (Obermiller et al., unpub. data, 1994), (3) Lanzo, Western Alps (Bodinier $e t$ al., 1991); for these peridotites, a radiogenic-growth correction (see text for explanation) has been applied, to make the data comparable with present-day MORB mantle and with the EL lherzolites.

Mercier (1980) are consistent with the transition from spinel- to plagioclase-facies stability field, i.e. depressurization.

Temperature estimates lead to two main conclusions: (1) the spinel-facies equilibration occurred at relatively low temperatures $\left(<1100^{\circ} \mathrm{C}\right)$, compatible with continental geothermal gradients; (2) the decompressional evolution from spinel- to plagioclasefacies stability field was accompanied by slight cooling, at temperatures significantly lower than the dry solidus. A similar trajectory has been obtained for the Erro-Tobbio peridotites (Hoogerduijn Strating et al., 1993), which are interpreted as fragments of subcontinental lithospheric mantle emplaced at shallow levels by tectonic denudation mechanisms, during the early stage of rifting of the JLPB.

\section{DISCUSSION}

\section{Amphibole metasomatism}

Disseminated Ti-pargasite is ubiquitous in all the subcontinental-mantle derived materials (Fabriès et al., 1991), and is generally considered to indicate metasomatic introduction of a volatile-rich melt or fluid phase (Menzies \& Hawkesworth, 1987; and references quoted therein) also enriched in incompatible elements. This evidence is based on trace element data of amphiboles crystallized in mantle peridotites (mainly documented in xenoliths), which are predominantly characterized by strong enrichment in incompatible elements. This amphibole metasomatism is commonly thought to modify the isotopic composition (especially the $\mathrm{Sr}$ isotope ratio) of the bulk peridotites as well as the clinopyroxenes.

Amphiboles stable in the spinel-facies assemblage of the EL peridotites have low contents of $\mathrm{Sr}$ and $\mathrm{Ba}$ (Fig. 9), and their REE patterns are LREE depleted, similar to the coexisting clinopyroxene (Fig. 7b). Also, the clinopyroxenes in amphibole-bearing peridotites (see Table 11) show MORB-type isotopic signatures, consistent with the other analysed samples. This indicates, in contrast to the above generalization, that amphibole metasomatism did not affect the clinopyroxene chemistry.

Amphibole formation without concomitant introduction of LREE and $\mathrm{Sr}$ has also been documented for spinel lherzolites from the Red Sea (Zabargad peridotites; Piccardo et al., 1993; Western Arabian xenoliths; Henjes-Kunst et al., 1990) and spinel lherzolite xenoliths from the Bartoy volcanoes of Siberia (Ionov et al., 1992). According to these workers, the above features suggest that the metasomatic agent from which amphibole crystallized was not enriched in incompatible elements, thus excluding alkaline melts or related high-density fluids.

The interpretation proposed for the Zabargad lherzolites (Piccardo et al., 1993) considers that the composition of $\mathrm{H}_{2} \mathrm{O}$-rich fluids in equilibrium with the upper mantle could have been originally depleted in LREE and Sr. If an originally enriched metasomatic agent is assumed, we suggest that the explanation for this apparently paradoxical behaviour lies in the chromatographic nature of infiltration metasomatism (Korzhinskii, 1968; Hofmann, 1972). This causes the metasomatic fronts of the solutes to lag behind the fluid front itself. This lag is zero only if the bulk partition coefficient between solid and fluid is zero, and it increases with increasing partition coefficients. The delay of the cation enrichment fronts is also proportional to the distance the fluid has travelled from its source. Therefore the effect is expected to be significant only if the metasomatic fluid has migrated for some distance through the mantle before precipitating the amphiboles found in the EL peridotites.

In this context, it is worth noting that the stability of amphibole in mantle peridotites implies temperatures $<1100^{\circ} \mathrm{C}$. If the fluid had traversed hotter mantle before encountering the peridotites sampled, it would not have precipitated amphibole, and the chromatographic effects described above would have had 'time and space' to develop. The $1100^{\circ} \mathrm{C}$ limit is 
also consistent with temperature estimates obtained for the spinel-facies assemblages $\left(1000-1050^{\circ} \mathrm{C}\right)$ and furnishes further constraints to the evidence of lowtemperature, lithospheric equilibration of the EL peridotites. In any case, we conclude that the composition of the amphiboles contains little information about the initial composition of the metasomatic fluid, if this fluid has travelled through a few kilometres of mantle rock.

\section{The nature of mantle protolith}

The EL peridotites are characterized by rather fertile composition, with only slight depletion in fusible components. The evidence for this is (1) the lherzolitic modal compositions (cpx up to $10-15 \%$ by volume), (2) the REE clinopyroxene patterns, showing only moderate LREE depletion, and (3) the high $\mathrm{Na}, \mathrm{Ti}, \mathrm{Sr}$ and $\mathrm{Zr}$ contents in clinopyroxenes and whole rocks. The fertile chemical signature is coupled with $\mathrm{Sr}$ and $\mathrm{Nd}$ isotopic composition typical of MORB mantle. Their ${ }^{147} \mathrm{Sm} /{ }^{144} \mathrm{Nd}$ ratios $(0 \cdot 232-$ 0.253 ) are also consistent with unmodified MORB mantle.

In spite of significant chemical heterogeneity observed in the subcontinental lithospheric mantle (Menzies \& Hawkesworth, 1987), rather fertile lherzolitic compositions have been found in other subcontinental, dry spinel lherzolites from orogenic massifs (e.g. Baldissero, Lherz, Lanzo N; Obermiller et al., unpub. data, 1994; Fabriès et al., 1989; Bodinier et al., 1991; Downes et al., 1991) and in mantle xenoliths from rift valley-mobile belt regions (Stosch et al., 1986; Stosch \& Lugmair, 1986; Menzies \& Hawkesworth, 1987; Galer \& O'Nions, 1989). These mantle rocks are also predominantly characterized by MORB isotopic signature, as is observed in the EL peridotites.

The above features indicate that the chemical and isotopic signature of EL peridotites is typical of many subcontinental lithospheric peridotites. A further implication is that large portions of subcontinental lithospheric mantle may be characterized by relatively homogeneous chemical and isotopic composition, typical of a MORB mantle source. Much of the subcontinental lithospheric mantle may therefore be similar to the MORB-type mantle, because it is derived by accretion of asthenospheric mantle to the lithosphere, and this lithosphere did not suffer, during geologic times, any significant metasomatic or melt infiltration process. This interpretation has been inferred for various subcontinental sp lherzolite xenoliths (Stosch et al., 1986; Ionov et al., 1992; and references therein) whose MORB isotopic signatures reflect a long-term depletion history before their incorporation into the lithosphere. For these rocks, estimates about the timing of depletion and consequent lithospheric accretion have been derived by model age calculations, combining the $\mathrm{Rb}-\mathrm{Sr}$ and $\mathrm{Sm}-\mathrm{Nd}$ systematics. These calculations generally yield Proterozoic ages.

Most of the analysed EL peridotites have ${ }^{147} \mathrm{Sm} /{ }^{144} \mathrm{Nd}$ of $0 \cdot 23-0 \cdot 25$, consistent with a typical depleted mantle (DM) source. Consequently, they do not contain age information about how long they have been incorporated in the lithosphere. For these samples, $\mathrm{Sr}$ and $\mathrm{Nd}$ model ages can be solely computed assuming a simple two-stage evolution from a chondritic mantle source (CHUR). Although this calculation gives apparently consistent ages between 1.7 and $1.9 \mathrm{Ga}$ (Table 11), this result has little geological age significance, because the depleted upper mantle appears to behave generally as an open system. This is clearly indicated by the systematics of $\mathrm{Pb}$ isotopes in MORB (Galer \& O'Nions, 1985; White, 1993). More useful information can be derived from sample ER-S2/2, whose very depleted isotopic composition allows us to calculate model ages assuming either a primitive or a depleted mantle source. The $\mathrm{Sr}$ model age from a DM source has been calculated assuming that the evolution of the depleted mantle reservoir started $4 \mathrm{Ga}$ ago with a value of ${ }^{87} \mathrm{Sr} /{ }^{86} \mathrm{Sr}=0.6996$. Nd model ages have been computed assuming different DM source compositions (Table 11; Fig. 11a), including a high ${ }^{43} \mathrm{Nd} /{ }^{144} \mathrm{Nd}_{\mathrm{DM}}$ value of 0.51335 as a maximum value for MORB (White, 1985) coupled with a low ${ }^{147} \mathrm{Sm} /{ }^{144} \mathrm{Nd}\left(0.2148 ;\right.$ Hart \& Zindler, 1986) $\left(\mathrm{DM}_{2}\right)$, to obtain a minimum $\mathrm{Nd}$ model age. The calculated model ages range between $2.4 \mathrm{Ga}$ and $780 \mathrm{Ma}$. In particular, the 1.2-Ga $\mathrm{Sr}$ age and the $780-\mathrm{Ma} \mathrm{Nd}$ age can be regarded as minimum ages of differentiation. These results point to a long-term depletion event for sample ER-S2/2. Thus, this single sample provides the strongest evidence that the EL peridotites are actually subcontinental lithosphere and that they were probably accreted to the lithosphere in Proterozoic time. This conclusion is further strengthened by the fact that such extremely depleted $\mathrm{Sr}$ isotopic compositions $\left({ }^{87} \mathrm{Sr} /{ }^{86} \mathrm{Sr}=\right.$ 0.701736; sample ER-S2/2) have never been observed in oceanic basalts.

Another remarkable geochemical feature of the EL peridotites, which could yield information about the nature of their mantle protolith, is the occurrence of positive $\mathrm{Eu}$ anomalies $\left(\mathrm{Eu} / \mathrm{Eu}^{*}=1 \cdot 13-1 \cdot 24\right)$ in the cores of spinel-facies porphyroclastic clinopyroxenes from Type A samples (ER-R3/3 and ERS2/2) (Fig. 7). This anomaly is also exhibited by coexisting spinel-facies amphiboles (sample ER-R3/3; 

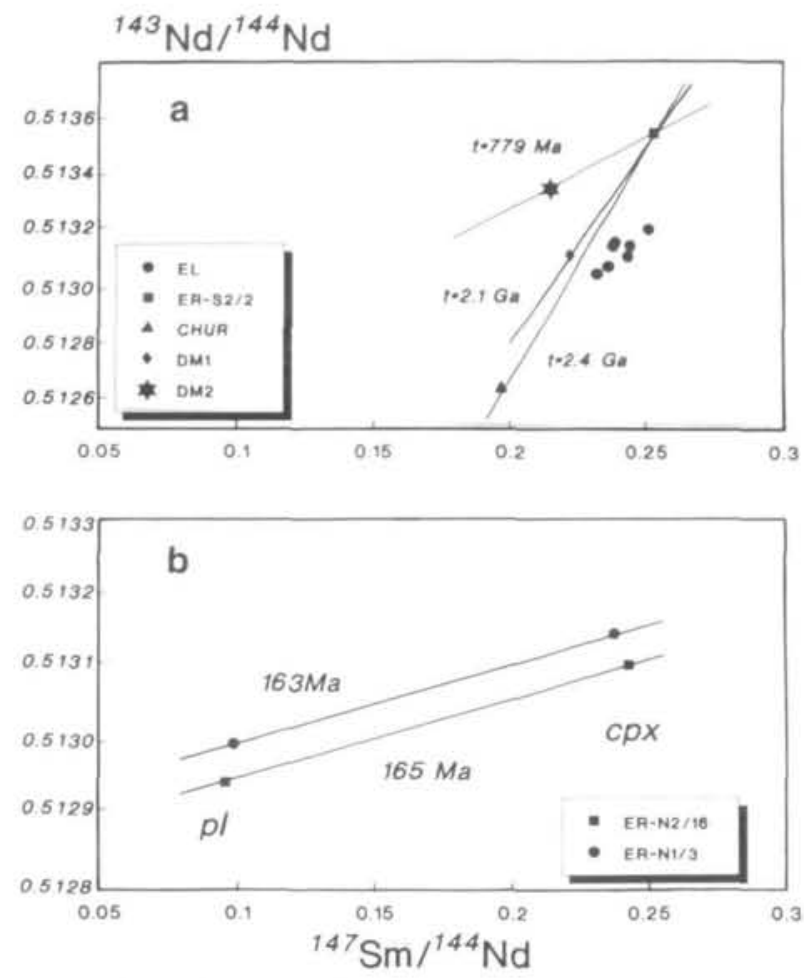

Fig. 11. ${ }^{143} \mathrm{Nd} /{ }^{144} \mathrm{Nd}$ vs ${ }^{147} \mathrm{Sm} /{ }^{144} \mathrm{Nd}$ diagram for: (a) EL clinopyroxene separates (see text and Table 11 for better explanation and definition of CHUR, DM 1 and $\mathrm{DM}_{2}$ ), and (b) clinopyroxene (cpx) and plagioclase (pl) separates from samples ER-N2/16 and ER-N1/3; the two samples define consistent isochrons of 163 and $165( \pm 20) \mathrm{Ma}$, respectively (see text for further explanations).

$\left.\mathrm{Eu} / \mathrm{Eu}^{*}=1 \cdot 18-1 \cdot 20\right)$. A simple explanation for this feature would be to assume an equilibration with a mineral phase able to compensate this $\mathrm{Eu}$ enrichment by a corresponding depletion. However, the analysed spinel-facies orthopyroxene does not show negative Eu anomalies (Fig. 7b), and olivine and spinel have REE contents too low to be considered in the mass balance. Moreover, it is evident that the positive $\mathrm{Eu}$ anomaly tends to disappear towards the rims of porphyroclasts and in plagioclase-facies granoblastic clinopyroxenes which, instead, show the progressive development of negative $\mathrm{Eu}$ anomalies, owing to the equilibrium crystallization with plagioclase (Rampone et al., 1993). This means that the Eu enrichment in spinel-facies clinopyroxene cannot be related to diffusive redistribution from plagioclase, as proposed by Henderson et al. (1976) and Seifert \& Chadima (1989). The plagioclase-facies re-equilibration, in fact, causes the opposite effect. Because there is no obvious mineral present in the rock (stable in the sp assemblage) which might compensate the positive Eu anomaly of the clinopyroxene with a complementary and sufficiently large negative anomaly, it is likely that the whole rocks also have positive anomalies (although we cannot be absolutely certain of this because we do not have the appropriate whole-rock analyses to demonstrate this conclusively). How such positive anomalies might originate in the mantle is not at all clear. Compensating negative $\mathrm{Eu}$ anomalies are sometimes found in garnets, for example in garnet lherzolites from the Alpe Arami and Monte Duria, central Alps (unpublished ion probe data). However, to generate a positive whole-rock anomaly, the garnet would have to be removed from the rock by some sort of disequilibrium melting process. Another way to create positive whole-rock $\mathrm{Eu}$ anomalies in mantle rocks might be equilibration with subducted crustal materials containing cumulus plagioclase, which would become unstable at mantle pressures but leave behind $\mathrm{Eu}$ anomalies as their geochemical 'fingerprint'. Such speculations gain some credibility by the observations from Hawaiian basalts, some of which have significant positive Eu anomalies, which have apparently been inherited from their mantle sources (e.g. Feigenson et al., 1983; West et al., 1992).

Alternatively, if we assume that no positive $\mathbf{E u}_{\mathbf{u}}$ anomaly exists in the bulk rock, we could speculate that the EL peridotites were formerly equilibrated in the garnet-facies stability field. During the sp-facies re-equilibration, most of clinopyroxenes preserved the Eu positive anomaly (recording their previous equilibration with garnet), whereas some clinopyroxenes (not analysed) crystallized as product of the garnet breakdown and inherited a compensating negative Eu anomaly. This garnet-facies equilibration could be related to the early (Proterozoic) upwelling of the EL peridotites from deeper asthenospheric mantle and consequent accretion to the lithosphere. More detailed ion probe investigations would be necessary to substantiate this hypothesis. We note, however, that a similar process, implying local geochemical disequilibrium during subsolidus partitioning, has been involved to explain the occurrence of unusual (garnet-like) trace element abundances in orthopyroxene and clinopyroxene from the Zabargad Al-Di pyroxenites (Vannucci et al., 1993b).

\section{The decompressional evolution during Triassic-Jurassic extension of the Ligurian Tethys}

The EL peridotites record a composite history of progressive deformation (from granular to tectonite fabrics) and recrystallization from spinel- to plagioclase-facies stability field. This subsolidus evolution is testified by major and trace element redistribution between mineral phases, such as (1) $\mathrm{Al}, \mathrm{Na}, \mathrm{Sr}$ and 
$\mathrm{Eu} / \mathrm{Eu}^{*}$ decrease, and $\mathrm{Y}, \mathrm{V}, \mathrm{Sc}, \mathrm{Cr}, \mathrm{Zr}$ and $\mathrm{Ti}$ increase in clinopyroxene, and (2) Al decrease, and $\mathrm{Cr}$ and $\mathrm{Ti}$ increase in spinel. Mass balance calculations (Rampone et al., 1993) demonstrate that the element redistribution occurred in response to the metamorphic reaction governing the transition from the spinel- to the plagioclase-facies assemblages. Thermometric estimates indicate that this decompressional evolution occurred under slightly decreasing temperature conditions (from 1000 $1100^{\circ} \mathrm{C}$ to $900^{\circ} \mathrm{C}$ ).

Plagioclase and clinopyroxene of two EL samples which were significantly recrystallized in plagioclasefacies conditions have been analysed for $\mathrm{Sr}$ and $\mathrm{Nd}$ isotopic compositions and $\mathrm{Sm}$ and $\mathrm{Nd}$ concentrations, to determine the age of this subsolidus pl-facies re-equilibration.

In both samples, clinopyroxene and plagioclase show the same ${ }^{87} \mathrm{Sr} /{ }^{86} \mathrm{Sr}$ ratio (Table 11 ), thus indicating $\mathrm{Sr}$ isotopic equilibrium. By contrast, they are characterized by different $\mathrm{Nd}$ isotopic compositions, in response to their different ${ }^{147} \mathrm{Sm} /{ }^{144} \mathrm{Nd}$ ratios (plagioclase: 0.095-0.098; clinopyroxene: $0.238-0.243$ ). This is shown in Fig. $11 \mathrm{~b}$, where the two plagioclase-clinopyroxene pairs define essentially parallel isochrons giving ages of 163 and 165 $\mathrm{Ma}( \pm 20 \mathrm{Ma})$.

Plagioclase-clinopyroxene isochrons are not used frequently because the $\mathrm{Sm} / \mathrm{Nd}$ ratios of these two minerals are not very different, and the resulting uncertainty on the calculated ages is comparatively large. Nevertheless, the good agreement between the two obtained ages and the relatively small analytical errors lend credibility to these isochrons and provide important information about the timing of EL peridotite subsolidus evolution. In particular, the 163$165 \mathrm{Ma}$ cooling age indicates that this event occurred slightly before the oceanic opening stage (Bathonian-Callovian) of the JLPB (Hoogerduijn Strating, 1991).

\section{Geodynamic implications}

The predominance of rather fertile lherzolites is a peculiar feature of the upper-mantle rocks early exposed on the sea-floor within the Jurassic Ligurian Piemontese basin (JLPB) (Bezzi \& Piccardo, 1971; Nicolas \& Jackson, 1972). These fertile lherzolites have been interpreted as (1) asthenospheric mantle which upwelled adiabatically and intruded into the continental lithosphere to produce the newly formed Jurassic oceanic sequences (Nicolas, 1984, 1986), or (2) lithospheric subcontinental mantle which was exposed on the sea-floor during the ocean opening by passive extensional mechanisms (Decandia \& Elter,
1969; Lombardo \& Pognante, 1982; Lemoine, 1983), following a subsolidus decompressional trajectory (Beccaluva et al., 1984). These two different interpretations have been applied to chemically and texturally similar lherzolites from different sectors of the Alpine chain.

The results of our petrologic and isotopic investigations indicate that the EL peridotites represent pre-Triassic subcontinental lithospheric mantle, presumably accreted to the lithosphere during Proterozoic time. Similar compositional features (i.e. a rather fertile character coupled with MORB isotopic signature) have been documented for other ophiolitic peridotites from the Alpine chain (e.g. Lanzo massif; Bodinier et al., 1991), as well as for peridotite bodies still linked to the Adria continental basement (e.g. Baldissero; Obermiller et al., unpub. data, 1994). It seems plausible, therefore, to interpret the above ophiolitic peridotites as representative of the pre-oceanic subcontinental mantle of this part of the Western Tethys. The same interpretation has been inferred for the Lanzo peridotite body, at least concerning its northern part (Bodinier et al., 1991).

Our petrologic and isotopic studies indicate, moreover, that the EL subcontinental mantle underwent an uplift history marked by a subsolidus evolution with slightly decreasing temperatures under decompression. This stage probably developed during the pre-oceanic rifting of the JLPB, in agreement with the 165-Ma cooling age obtained for the re-equilibration under plagioclase-facies conditions.

The above evolutionary features, and in particular the Proterozoic model age of one sample, are consistent with a passive uplift of the subcontinental lithosphere, rather than with an active and adiabatic upwelling of the asthenosphere. Thermal modelling (Ruppel et al., 1988) for rocks exhumed via passive (pure and/or simple shear) mechanisms suggests that, in the case of simple (i.e. asymmetric) shear, rocks follow almost linear $P-T$ paths with progressively decreasing temperature and pressure when they evolve as the footwall of a low-angle normal fault.

A consistent petrological and structural evolution has been documented for the ophiolitic peridotites of the Erro-Tobbio body (Ligurian Alps; Piccardo et al., 1990; Vissers et al., 1991; Hoogerduijn Strating et al., 1993). This subsolidus evolution has been related to the rifting stage in the JLPB, leading to tectonic unroofing of the lithospheric upper mantle by means of passive and asymmetric extension of the lithosphere (Wernicke, 1985). This geodynamic process is regarded as the most plausible for the pre-oceanic rift in the JLPB (Lemoine et al., 1987; Dal Piaz, 
1993), and is thought to be responsible for the exposure of the lithospheric subcontinental mantle during the inception of the oceanic basin (Piccardo et al., 1992).

\section{GONGLUSIONS}

\section{The EL peridotite evolution}

The External Liguride peridotites represent a slice of subcontinental lithospheric mantle which was exposed on the sea-floor during early stages of oceanic opening of the Ligurian Piemontese basin. Chemical and isotopic compositions highlight their similarity to relatively fertile MORB-type mantle sources. The MORB isotopic signature is documented for various orogenic spinel lherzolites of subcontinental lithospheric provenance, at present outcropping in the Alpine-Apennine chain (Richard \& Allègre, 1980; Bodinier et al., 1991; Obermiller et al., unpub. data, 1994). This could indicate that part of the subcontinental lithospheric mantle of the Western Tethys was derived through early (Proterozoic) accretion of MORB-type asthenospheric mantle.

The history of the External Liguride peridotites can be summarized as follows:

(1) ancient (presumably Proterozoic) upwelling and depletion of an asthenospheric MORB-type mantle;

(2) lithospheric accretion and annealing recrystallization (at $T<1100^{\circ} \mathrm{C}$ ) in the spinel-facies stability field;

(3) modal metasomatism (crystallization of kaersutitic-Ti-pargasitic amphibole) by means of hydrous fluids not enriched in incompatible elements. A chromatographic mechanism is considered to account for the low concentration of LREE, Sr and $\mathrm{Ba}$ in the reacting fluid;

(4) Triassic-Jurassic decompressional evolution recorded by progressive deformation (from granular to tectonite-mylonite types) and metamorphic reequilibration from spinel- to plagioclase-facies stability field (plagioclase-clinopyroxene cooling ages of $\sim 165 \mathrm{Ma}$ );

(5) shallow emplacement, recorded by the intrusion of MORB-type chilled basaltic dykes and lowgrade alteration processes (peridotite serpentinization and basic dyke rodingitization).

Early Jurassic decompressional evolution recorded by the EL peridotites is consistent with uplift by means of denudation in response to asymmetric lithospheric extension. This is considered to be the most likely geodynamic mechanism to account for the exposure of huge bodies of subcontinental litho- spheric mantle during early stages of opening of an oceanic basin.

\section{AGKNOWLEDGEMENTS}

E. Rampone thanks R. Petrini and C. Quercioli (CNR, Istituto di Geocronologia e Geochimica Isotopica, Pisa) for availability of the mineral separation laboratory, and I. Raczek (Max-PlanckInstitut, Mainz) for assistance with isotopic analyses. E. H. Strating and G. W. Ernst are thanked for reviewing the manuscript. Funding was provided by the Italian MURST and CNR, in the framework of the project 'Evolution of the lithosphere: deep structure and metamorphism'.

\section{REFERENGES}

Abbate, E., Bortolotti, V., Passerini, V. \& Sagri, M., 1970. Introduction to geology of the Northern Apennines. Sedimentary Geology 4, 207-249.

Anders, E. \& Ebihara, M., 1982. Solar system abundances of the elements. Geochimica el Cosmochimica Acta 46, 2363-2380.

Basu, A. R., 1978. Trace elements and Sr-isotopes in some mantlederived hydrous minerals and their significance. Geochimica at Cosmochimica Acta 42, 659-668.

Beccaluva, L., Macciotta, G., Piccardo, G. B. \& Zeda, O., 1984. Petrology of lherzolitic rocks from the Northern Apennine ophiolites. Lithos 17, 299-316.

Beccaluva, L., Piccardo, G. B. \& Serri, G., 1980. Petrology of Northern Apennine ophiolites and comparison with other Tethyan ophiolites. Proceedings International Ophiolite Symposium, Cyprus, pp. 314-331.

Bence, A. E. \& Albee, A. L., 1968. Empirical correction factors for the electron microanalysis of silicates and oxides. Foumal of Geology 76, 382-403.

Bezzi, A. \& Piccardo, G. B., 1971. Structural features of the Ligurian ophiolites: petrologic evidence for the ocean floor of the northern Apennine geosyncline. A contribution to the problem of the Alpine-type gabbro-peridotite associations. Memorie della Sacietá Geologica Italiana 10, 55-63.

Bodinier, J.-L., Dupuy, C. \& Dostal, J., 1988. Geochemistry and petrogenesis of Eastern Pyrenean peridotites. Geochimica et Cosmochimica Acta 52, 2893-2907.

Bodinier, J. L., Guiraud, M., Fabriès, J. \& Dupuy, C., 1987. Petrogenesis of layered pyroxenites from the Lherz, Freychinede and Prades ultramafic bodies (Ariège, French Pyrenees). Geochimica el Cosmochimica Acta 51, 279-290.

Bodinier, J. L., Menzies, M. A. \& Thirlwall, M. F., 1991. Continental to oceanic mantle transition-REE and Sr-Nd isotopic geochemistry of the Lanzo Lherzolite Massif. In: Menzies, M. A., Dupuy, C. \& Nicolas, A. (eds) Orogenic Lherzolites and Mantle Processes. Fournal of Petrology, Special Lherzolite Issue, 191-210.

Bonatti, E. \& Michael, P. J., 1989. Mantle peridotites from continental rifts to ocean basins to subduction zones. Earth and Planetary Science Letters 91, 297-311.

Bottazzi, P., Ottolini, L. \& Vannucci, R., 1991. Determination of rare earth elements in sixteen silicate reference samples by secondary ion mass spectrometry using conventional energy filtering technique. Geostandards Newsletter 15(N.1), 51-57. 
Dal Piaz, G. V., 1993. Evolution of Austro-Alpine and upper Pennine basement in the North-Western Alps from Variscan convergence to post-Variscan extension. In: Von Raumer, J. F. \& Neubauer, F. (eds) Pre-Mesozoic Geology in the Alps. Berlin: Springer-Verlag, pp. 327-344.

Decandia, F. A. \& Elter, P., 1969. Riflessioni sul problema delle ofioliti nell'Appennino Settentrionale (nota preliminare). Atti della Societá Toscana di Scienze Naturali Residente in Pisa Memorie, Serie $A, 75,1-9$.

Dick, H. J. B., 1989. Abyssal peridotites, very low spreading ridge and ocean ridge magmatism. In: Saunders, A. D. \& Norry, M. J. (eds) Magmatism in the Ocean Basins, pp. 71-106.

Downes, H., Bodinier, J. L., Thirlwall, M. F., Lorand, J. P. \& Fabriès, J., 1991. REE and Sr-Nd isotopic geochemistry of the Eastern Pyrenean peridotite massifs: subcontinental lithospheric mantle modified by continental magmatism. In: Menzies, $M$ A., Dupuy, C. \& Nicolas, A. (eds) Orogenic Lherzolites and Mantle Processes. Journal of Petrology, Special Lherzolite Issue, 97-116.

Eberhardt, P., Ferrara, G. \& Tongiorgi, E., 1962. Determination de l'age des granites allochtones de l'Apennin septentrional. Bulletin de la Société Géologique de France 4, 666-667.

Elter, P. \& Raggi, G., 1965. Contributo alla conoscenza dell'Appennino Ligure 3. Tentativo di interpretazione delle brecce ofiolitiche cretaccee in relazione con movimenti orogenetici dell'Appennino I.igure. Bollettino della Societá Geologico Italiana 84, 1-14.

Ernst, W. G. \& Piccardo, G. B., 1979. Petrogenesis of some Ligurian peridotites: I. Mineral and bulk rock chemistry. Geochimica et Cosmochimica Acta 43, 219-237.

Fabriès, J., Bodinier, J.-L., Dupuy, C., Lorand, J.-P. \& Benkerrou, G., 1989. Evidence for modal metasomatism in the orogenic spinel lherzolite body from Cassou (Northeastern Pyrenees, France). Journal of Pelrology 30, 199-228.

Fabriès, J., Lorand, J.-P., Bodinier, J.-L. \& Dupuy, C., 1991 Evolution of the upper mantle beneath the Pyrenees: evidence from orogenic spinel lherzolite massifs. In: Menzies, M. A. Dupuy, C. \& Nicolas, A. (eds) Orogenic Lherzolites and Mantle Processes. Fournal of Petrology, Special Lherzolite Issue, 55-76.

Faure, G., 1986. Principles of Isolope Geology. New York: John Wiley.

Feigenson, M. D., Hofmann, A. W. \& Spera, F. J., 1983. Case studies on the origin of basalts. II. The transition from tholeiitic to alkalic volcanism on Kohala volcano, Hawaii. Contributions to Mineralogy and Petrology 84, 390-405.

Frey, F. A. \& Prinz, M., 1978. Ultramafic inclusions from San Carlos, Arizona: petrological and geochemical data bearing on their petrogenesis. Earth and Planetary Science Letters 38, 129-176.

Frey, F. A., Shimizu, N., Leinbach, A., Obata, M. \& Takazawa, E., 1991. Compositional variations within the lower layered zone of the Horoman peridotite, Hokkaido, Japan: constraints on models for melt-solid segregation. In: Menzies, M. A., Dupuy, C. \& Nicolas, A. (eds) Orogenic Lherzolites and Mantle Processes. Journal of Petrology, Special Lherzolite Issue, 211-227.

Frey, F. A., Suen, C. J. \& Stockman, H. W., 1985. The Ronda high temperature peridotite: geochemistry and petrogenesis. Geochimica el Cosmochimica Acta 49, 2469-2491.

Galer, S. J. G. \& O'Nions, R. K., 1985. Residence time of thorium, uranium and lead in the mantle with implications for mantle convection. Nature 316, 778-782.

Galer, S. J. G. \& O'Nions, R. K., 1989. Chemical and isotopic studies of ultramafic inclusions from the San Carlos Volcanic field, Arizona: a bearing on their petrogenesis. Journal of Petrology 30, 1033-1064.
Grauch, R. I., 1989. Rare earth elements in metamorphic rocks. In: Lipin, B. R. \& McKay, G. A. (eds) Geochemistry and Mineralogy of Rare Earth Elements. Mineralogical Society of America, Reviews in Mineralogy 21, 147-168.

Hart, S. R. \& Zindler, A., 1986. In search for a bulk-earth composition. Chemical Geology 57, 247-267.

Henderson, P., Fishlock, S. J., Laul, J. C., Cooper, T. D., Conrad, R. L., Boynton, W. V. \& Schmitt, R. A., 1976. Rare earth element abundances in rocks and minerals from the Fiskenaesset complex, West Greenland. Earth and Planetary Science Letters 30, $37-49$.

Henjes-Kunst, F., Altherr, R. \& Baumann, A., 1990. Evolution and composition of the lithospheric mantle underneath the Western Arabian peninsula: constraints from $\mathrm{Sr}-\mathrm{Nd}$ isotope systematics of mantle xenoliths. Contributions to Mineralogy and Petrology 105, 460-472.

Hofmann, A. W., 1972. Chromatographic theory of infiltration metasomatism and its application to feldspars. American foumal of Science 272, 69-90.

Hofmann, A. W., 1988. Chemical differentiation of the Earth: the relationship between mantle, continental crust, and oceanic crust. Earth and Planetary Science Letters 90, 297-314.

Hoogerduijn Strating, E. H., 1991. The evolution of the Piemonte-Ligurian ocean. A structural study of ophiolite complexes in Liguria (NW Italy). Ph.D. Thesis, University of Utrecht.

Hoogerduijn Strating, E. H., Piccardo, G. B., Rampone, E., Scambelluri, M. \& Visser, R. L. M., 1990. The structure and petrology of the Erro-Tobbio peridotite (Voltri Massif, Ligurian Alps): a two-day excursion with emphasis on processes in the upper mantle. Ofioliti 15, 119-184.

Hoogerduijn Strating, E. H., Rampone, E., Piccardo, G. B. Drury, M. R. \& Vissers, R. L. M., 1993. Subsolidus emplacement of mantle peridotites during incipient oceanic rifting and opening of the Mesozoic Tethys (Voltri Massif, NW Italy). Journal of Petrology 34, 901-927.

Ionov, D., Kramm, U. \& Stosch, H. G., 1992. Evolution of the upper mantle beneath the southern Baikal zone: a Sr-Nd isotope study of xenoliths from the Bartoy volcanoes. Contributions to Mineralogy and Pelrology 111, 235-247.

Ito, E., White, W. H. \& Gopel, C., 1987. The O, Sr, Nd and Pb isotope geochemistry of MORB. Chemical Geology 62, 157-176.

Jagoutz, E., Carlson, R. W. \& Lugmair, G. W., 1980. Equilibrated $\mathrm{Nd}$-unequilibrated $\mathrm{Sr}$ isotopes in mantle xenoliths. Noture 286, 708-710.

Jagoutz, E., Palme, H., Baddenhausen, H., Blum, K., Cendales, M., Dreibus, G., Spettel, B., Lorenz, V. \& Wanke, H., 1979. The abundance of major, minor and trace elements in the earth's mantle derived from ultramafic nodules. Proceedings of the 10th Lunar and Planetary Science Conference, Geochimica et Cosmochimica Acia 2 (Supplement II), 2031-2050.

Korzhinskii, D. S., 1968. The theory of metasomatic zoning. Mineralium Deposita 3, 222-231.

Leake, B. E., 1978. Nomenclature of amphiboles. Bulletin of Mineralogy 101, 453-468.

Lemoine, M., 1983. Serpentinite oceanic bottom in south Queyras ophiolite (French Western Alps): record of the incipient oceanic opening of the Mesozoic Ligurian Tethys. Eclogae Geologicae Helvetiae 76, 611-629.

Lemoine, M., Bas, T., Arnaud-Vanneau, A., Arnaud, H., Dumont, T., Gidon, M., Bourbon, M., de Graciansky, P. C., Rudkiewicz, J. L. \& Tricart, P., 1986. The continental margin 
of the Mesozoic Tethys in the Western Alps. Marine Petrology and Geology 3, 178-199.

Lemoine, M., Tricart, P. \& Boillot, G., 1987. Ultramafic and gabbroic ocean floor of the Ligurian Tethys (Alps, Corsica, Apennines): in search of a genetic model. Geology 15, 622-625.

Lombardo, B. \& Pognante, U., 1982. Tectonic implications in the evolution of the Western Alps ophiolite metagabbros. Ofioliti 7 , 371-394.

Lugovic, B., Altherr, R., Raczeck, I., Hofmann, A. W. \& Majer, V., 1991. Geochemistry of peridotites and mafic igneous rocks from Central Dinaric Ophiolite Belt, Yugoslavia. Contributions to Mineralogy and Petrology 106, 201-216.

Marcucci, M. \& Passerini, P., 1991. Radiolarian-bearing siliceous sediments in the Mesozoic of the Northern and Central Apennines. Ofioliti 16, 121-126.

McKay, G. A., 1989. Partitioning of rare earth elements between major silicate minerals and basaltic melts. In: Lipin, B. R. \& McKay, G. A. (eds) Geochemistry and Mineralogy of Rare Earth Elements. Mineralogical Society of America, Reviews in Mineralogy 21, 45-78.

Menzies, M. A. \& Dupuy, C., 1991. Orogenic massifs: protolith, process and provenance. In: Menzies, M. A., Dupuy, C. \& Nicolas, A. (eds) Orogenic Lherzolites and Mantle Processes. Journal of Petrology, Special Lherzolite Issue, 1-16.

Menzies, M. A. \& Hawkesworth, C. J., 1987. Upper mantle processes and composition. In: Nixon, P. H. (ed.) Mantle Xenoliths. Chichester: John Wiley, pp. 725-738.

Mercier, J. C. C., 1980. Single pyroxene thermobarometry. Tectonophysics 70, 1-37.

Nicolas, A., 1984. Lherzolites of the Western Alps: a structural review. In: Kornprobst, J. (ed.) 5th Intemational Kimberlite Conference Proceedings. Amsterdam: Elsevier, pp. 335-345.

Nicolas, A., 1986. Structure and petrology of peridotites: clues to their geodynamic environment. Reviews of Geophysics 24, 875895.

Nicolas, A. \& Jackson, E. D., 1972. Reparation en deux provinces des peridotites des chaînes alpines longeant la Méditerranée: implications géotectoniques. Bulletin Suisse de Minéralogie et Pétrographie 52(3), 479-495.

O'Reilly, S. Y., Griffin, W. L. \& Ryan, C. G., 1991. Residence of trace elements in metasomatized spinel lherzolite xenoliths: a proton-microprobe study. Contributions to Mineralogy and Petrology 109, 98-113.

Ottonello, G., Piccardo, G. B. \& Joron, J. L., 1984. Rare earth and 3d transition element geochemistry of peridotitic rocks: II. Ligurian peridotites and associated basalts. Fournal of Petrology 25, 379-393.

Pagani, G., Papani, G., Rio, D., Torelli, L., Zanzucchi, G. \& Zerbi, M., 1972. Osservazioni sulla giacitura delle ofioliti delle alte valli del T. Ceno e del F. Taro. Memorie della Societá Geologica Italiana 11, 531-546.

Palme, H. \& Nickel, K. G., 1985. Ca/Al ratio and composition of the Earth's upper mantle. Geochimica et Cosmochimica Acta 49, 2123-2132.

Piccardo, G. B., 1977. Le ofioliti dell'areale ligure: petrologia ed ambiente geodinamico di formazione. Rendiconti della Societa Italiana di Mineralogiá e Petrologia 33, 221-252.

Piccardo, G. B., Messiga, B. \& Vannucci, R., 1988. The Zabargad peridotite-pyroxenite association: petrological constraints on its evolution. Tectonophysics 150, 135-162.

Piccardo, G. B., Rampone, E. \& Vannucci, R., 1990. Upper mantle evolution during continental rifting and ocean formation: evidence from peridotite bodies of the Western Alpine-
Northern Apennine system. Memoires de la Société Géologique de France 156, 323-333.

Piccardo, G. B., Rampone, E. \& Vannucci, R., 1992. Ligurian peridotites and ophiolites: from rift to ocean formation in the Jurassic Ligure-Piemontese basin. Acta Vulcanologica 2, 313-325. Piccardo, G. B., Rampone, E., Vannucci, R., Shimizu, N., Ottolini, L. \& Bottazzi, P., 1993. Mantle processes in the subcontinental lithosphere: the case study on the rifted sp-lherzolites of the Zabargad Island (Northern Red Sea). European fournal of Mineralogy 5, 1039-1056.

Polvé, M. \& Allègre, C. J., 1980. Orogenic lherzolite complexes studied by ${ }^{87} \mathrm{Rb}-{ }^{87} \mathrm{Sr}$ : a clue to understand the mantle convection processes? Earth and Planetary Science Letters 51, 71-93.

Rampone, E., 1987. Petrology of basaltic dykes from the Mt. Aiona ultramafics (External Ligurides, northern Apennine, Italy). Ofioliti 12, 507-516.

Rampone, E., 1992. Studio petrologica e geochimico delle peridotiti dell'Appennino settentrionale. Ph.D. Thesis, University of Genova.

Rampone, E., Bottazzi, P. \& Ottolini, L., 1991. Complementary $\mathrm{Ti}$ and $\mathrm{Zr}$ anomalies in orthopyroxene and clinopyroxene from mantle peridotites. Nature 354, 518-521.

Rampone, E., Piccardo, G. B., Vannucci, R., Bottazzi, P. \& Ottolini, L., 1993. Subsolidus reactions monitored by trace element partitioning: the spinel- to plagioclase-facies transition in mantle peridotites. Contributions to Mineralogy and Petrology 115, 117.

Reisberg, L. \& Zindler, A., 1987. Extreme isotopic variations in the upper mantle: evidence from Ronda. Earth and Planetary Science Letters 81, 29-45.

Reisberg, L., Zindler, A. \& Jagoutz, E., 1989. Further Sr and Nd isotopic results from peridotites of the Ronda Ultramafic Complex. Earth and Planetary Science Letters 96, 161-180.

Richard, P. \& Allègre, C. J., 1980. Neodymium and strontium isotope study of ophiolite and orogenic lherzolite petrogenesis. Earth and Planetary Science Letters 47, 65-74.

Ringwood, A. E., 1975. Composition and Petrology of the Earth's Mantle. New York: McGraw-Hill, $618 \mathrm{pp}$.

Rivalenti, G., Garui, G., Rossi, A. \& Sinigoi, S., 1979. Spinels as petrogenetic indicators of the Ivrea-Verbano basic complex (Italian Western alps). Whole rock geochemistry. Bollettino della Societí Geologica Italiana 94, 1149-1186.

Roden, M. K., Frey, F. A. \& Francis, D. M., 1984. An example of consequent mantle metasomatism in peridotite inclusion from Nunivak, Alaska. Joumal of Petrology 25, 546-577.

Roden, M. K., Irving, A. J. \& Murthy, V. R., 1988. Isotopic and trace element composition of the upper mantle beneath a young continental rift: results from Kilbourne Hole, New Mexico. Geochimica et Cosmochimica Acta 52, 461-474.

Ruppel, C., Royden, L. \& Hodges, K. V., 1988. Thermal modeling of extensional tectonics: applications to pressure-temperature-time histories of metamorphic rocks. Tectonics 7, 947-957.

Sachtleben, T. \& Seck, H. A., 1981. Chemical control of Al-solubility in orthopyroxenes and its implications on pyroxene geothermometry. Contributions to Mineralogy and Petrology 78, 156165.

Seifert, K. E. \& Chadima, S. A., 1989. Depletion of heavy rareearth elements in metamorphic minerals from Adirondack anorthosites. Geology 17, 1004-1006.

Shimizu, N., Semet, M. P. \& Allègre, J. C., 1978. Geochemical applications of quantitative ion microprobe analysis. Geochimica et Cosmochimica Acta 42, 1321-1334. 
Stosch, H. G., 1982. Rare earth element partitioning between minerals from anhydrous spinel peridotite xenoliths. Geochimica et Cosmochimica Acta 46, 793-811.

Stosch, H. G., Carlson, R. W. \& Lugmair, G. W., 1980. Episodic mantle differentiation: $\mathrm{Nd}$ and $\mathrm{Sr}$ isotopic evidence. Earth and Planetary Science Letters 47, 263-271.

Stosch, H. G. \& Lugmair, W. G., 1986. Trace element and $\mathrm{Sr}$ and $\mathrm{Nd}$ isotope geochemistry of peridotite xenoliths from the Eifel (West Germany) and their bearing on the evolution of the subcontinental lithosphere. Earth and Planetary Science Letters 80, 281298.

Stosch, H. G., Lugmair, W. G. \& Kovalenko, V. I., 1986. Spinel peridotite xenoliths from the Tariat Depression, Mongolia. II Geochemistry and $\mathrm{Nd}$ and $\mathrm{Sr}$ isotopic composition and their implications for the evolution of the subcontinental lithosphere. Geochimica et Cosmochimica Acta 50, 2601-2614.

Stosch, H. G. \& Seck, H. A., 1980. Geochemistry and mineralogy of two spinel peridotite suites from Dreiser Weiher, West Germany. Geochimica et Cosmochimica Acta 44, 457-470.

Vannucci, R., Rampone, E., Piccardo, G. B., Ottolini, L. \& Bottazzi, P., 1993a. Ophiolitic magmatism in the Ligurian Tethys: an ion microprobe study of basaltic clinopyroxenes. Contributions to Mineralogy and Petrology 115, 123-137.

Vannucci, R., Shimizu, N., Bottazzi, P., Ottolini, L., Piccardo, G. B. \& Rampone, E., 1991. REE and HFSE geochemistry of mantle clinopyroxenes from the Zabargad peridotite-pyroxcnite association. In: Menzies, M. A., Dupuy, C. \& Nicolas, A. (eds) Orogenic Lherzolites and Mantle Processes. Journal of Petrology, Special Lherzolite Issue, 255-270.

Vannucci, R., Shimizu, N., Piccardo, G. B., Ottolini, L. \& Bottazzi, P., 1993b. Distribution of trace elements during breakdown of mantle garnet: an example from Zabargad. Contributions to Mineralogy and Petrology 113, 437-449.
Vissers, R. L. M., Drury, M. R., Hoogerduijn Strating, E. H. \& van der Wal, D., 1991. Shear zones in the upper mantle: a case study in an Alpine therzolite massif. Geology 19, 990-993.

Voshage, H., Sinigoi, S., Mazzuchelli, M., Demarchi, G., Rivalenti, G. \& Hofmann, A. H., 1988. Isotopic constraints on the origin of ultramafic and mafic dykes in the Balmuccia peridotite (Ivrea Zone). Contributions to Mineralogy and Petrology 100, 261-267.

Wells, P. R. A., 1977. Pyroxene thermometry in simple and complex systems. Contributions to Mineralogy and Petrology 62, 129-139.

Wernicke, B., 1985. Uniform sense normal simple shear of the continental lithosphere. Canadian Joumal of Earth Sciences 22, 108-125.

West, H. B., Garcia, M. O., Gerlach, D. C. \& Romano, J., 1992. Geochemistry of tholeiites from Lanai, Hawaii. Contributions to Mineralogy and Petrology 112, 520-542.

White, W. M., 1985. Sources of oceanic basalts: radiogenic isotope evidence. Geology 13, 115-118.

White, W. M., 1993. ${ }^{283} \mathrm{U} /{ }^{204} \mathrm{~Pb}$ in MORB and open system evolution of the depleted mantle. Earth and Planetary Science Letters 115, 211-226.

White, W. M. \& Patchett, P. J., 1984. HF-Nd-Sr isotopes and incompatible element abundances in island arcs: implications for magma origins and crust-mantle evolution. Earth and Planetary Science Letters 67, 167-185.

Wood, B. J. \& Banno, S., 1973. Garnet-orthopyroxene and orthopyroxene-clinopyroxene relations in simple and complex systems. Contributions to Mineralogy and Petrology 42, 109-124.

Zindler, A. \& Hart, S. R., 1986. Chemical geodynamics. Annual Review of Earth and Planetary Sciences 14, 493-571.

RECEIVED 3 NOVEMBER 1993 REVISED TYPESCRIPT ACCEPTED 3 MAY 1994 